\title{
Fast Parking Control of Mobile Robots: A Motion Planning Approach With Experimental Validation
}

\author{
Ti-Chung Lee, Chi-Yi Tsai, and Kai-Tai Song, Associate Member, IEEE
}

\begin{abstract}
This paper presents a solution to the general parking problem of nonholonomic mobile robots based on motion planning and tracking controller design. A new global tracking controller is first proposed to achieve global uniformly asymptotic stability and local exponential convergence. The parking problem is then transformed into a tracking one by adding a redesigned virtual trajectory to the original trajectory, thus guaranteeing practical stability with exponential convergence. Further improvement in parking performance is obtained through linearization and pole-placement methods. One feature of our approach is that fast convergence in parking and tracking can be treated at the same time without switching between two controllers. Moreover, a tuning function is used to enhance parking performance. With the proposed framework, various tracking controllers given in the literature can be adopted to handle parking problems. The effectiveness of the proposed methods is verified by several interesting experiments including parallel parking and back-into-garage parking.
\end{abstract}

Index Terms-Locally exponential convergence, mobile robots, motion planning, parking control, pole placement, tracking control.

\section{INTRODUCTION}

$\mathbf{M}$ OBILE robot tracking control problems have been studied extensively in recent decades [15], [20], [21], [34]. Reported controllers are usually simple and can achieve fast tracking control. In general, getting a nonholonomic mobile robot to track a moving trajectory (the tracking problem) is often easier than getting it to stop at a specified location (the parking problem) [26]. In fact, it is well known that according to Brockett's theorem [3], [30], the parking problem cannot be solved by employing continuous time-invariant feedback. To overcome this shortcoming and solve the regulation (parking) problem for general nonholonomic systems, including unicycle-modeled mobile robots, several research directions have been tried using nonlinear control approaches [1], [2], [4]-[7], [9]-[13], [17], [18], [21]-[25], [29], [30], [32], [33], [36]. For detailed discussions and comments on recent developments, see [21].

Manuscript received February 21, 2003; revised November 6, 2003. Manuscript received in final form January 14, 2004. Recommended by Associate Editor K. Moore. This work was supported by the National Science Council of Taiwan, R.O.C., under Grants NSC 90-2213-E-009-023, NSC 90-2213-E-009-027, and NSC-91-2213-E-159-004.

T.-C. Lee is with the Department of Electrical Engineering, Ming Hsin University of Science and Technology, Hsinchu 304, Taiwan, R.O.C. (e-mail: tc1120@ms19.hinet.net)

C.-Y. Tsai and K.-T. Song are with the Department of Electrical and Control Engineering, National Chiao Tung University, Hsinchu 300, Taiwan, R.O.C (e-mail: u9112824@cn.nctu.edu.tw; ktsong@cc.nctu.edu.tw).

Digital Object Identifier 10.1109/TCST.2004.826964
Recently, research interest has centered on improving tracking performance, especially in the areas of convergence rate and robustness. It is well known that smooth feedback cannot achieve an exponential convergence rate [23]. Thus, most of the exponential stabilizers in the current literature employ either discontinuous feedback laws [1] or continuous but not differentiable functions [6], [23], [24], [36]. Another approach adopts some kind of linearization technique to guarantee exponential convergence to within any desired small radius of the origin [4], [18]. In [22], an interesting example was proposed to show that although the continuous homogeneous feedback laws proposed in recent literature can achieve exponential convergence, they are not robust w.r.t. system uncertainty in general. Because of this, problems concerning robust control have recently attracted much research attention [13], [14], [18].

Despite the apparent advances represented by the methods mentioned above, there remain some major restrictions on their applications as reflected in current literature. For example, these methods cannot be applied to cases in which the tracking trajectory is not directly to a point as in, for instance, the parallel parking and the back-into-garage problems. In such cases, switching between two different types of controller is necessary. To overcome this drawback, a single controller was proposed to treat tracking and parking problems simultaneously in [11], [12], and [21]; however, it was observed that the convergence rate became very slow when the mobile robot did not exactly follow the desired trajectory before stopping. This happened because the proposed controllers used smooth feedback and such controllers usually have poor convergence behavior [21]. Solving the general parking problem when the tracking trajectory may be not be just a point, and guaranteeing rapid convergence at the same time is still an interesting problem in the area of nonlinear control systems.

Quite recently, novel approaches to solving the general tracking problem, including the parking case, were proposed in [10] and [26]. These approaches achieved practical stability with rapid convergence, rather than asymptotic stability. In contrast to these results, Samson et al. proposed a motion-planning approach to solving the regulation problem in [33]. They used a virtual moving trajectory to transform the regulation problem into a tracking-control problem. An interesting example and simulation result were presented in [32]. However, complete analysis on the stability and performance, notably in the area of convergence rate and robustness, were not realized and reported in these studies.

This paper continues the research line suggested in [32] and [33]. It proposes a new controller design for the general parking problem based on a more precise form of motion 
planning. Indeed, a new global tracking controller is first proposed. A novel stability analysis is given to show that the origin of the closed-loop system is uniformly globally asymptotically stable and locally exponentially stable under certain persistent excitation (PE) conditions. Furthermore, the parking problem is transformed into tracking one by addition of a redesigned virtual trajectory to the original trajectory, thus guaranteeing practical stability with exponential convergence via the proposed tracking controller. The linearization and pole-placement method proposed in [18] is used to improve parking performance including those of transient behavior, convergence rate, and robustness. In particular, the tracking and parking problems can be solved and rapid convergence achieved simultaneously by means of a single tracking controller. Moreover, a predesigned tuning function $k_{2}(t)$, employed in the proposed control scheme, provides a smooth transition from moving to parking. The proposed approach can be extended to other higher order nonholonomic systems, for example, in the parking control of underactuated ships [19]. Furthermore, with the proposed framework, various tracking controllers given in the literature can also be adopted to handle the parking problem.

The effectiveness of the proposed controllers is first verified using computer simulation. The simulation results are further verified via several interesting experiments including parallel parking and back-into-garage parking. Although not designed to explore certain advanced theoretical aspects of the controller, the experimental results do reveal some positive features of the design. We show that the proposed controller can overcome tracking errors caused by imperfections in the practical system such as quantization and sensor errors. This indicates that the proposed controller possesses a certain degree of robustness. Moreover, we compare the proposed parking controller with the saturation feedback controller proposed in [21], and our experimental results show that the controller proposed here also enhances parking performance. One interesting issue is the role of the designed tuning function $k_{2}(t)$. Experimental results show that the tuning function facilitates a smooth transformation from tracking mode to parking mode. The experimental results also suggest possible application of the proposed parking controller to the docking of autonomous mobile robots or home robots.

The rest of this paper is organized as follows. Section II describes formulation of the parking problem. Section III presents the main results of our parking controller design. Section IV reports on simulations and experimental results from the proposed controller. A practical realization employing a mobile laboratory robot is illustrated. Extended discussion of several interesting experimental observations is presented. We conclude in Section V.

\section{From Tracking to PARKING CONTROL}

\section{A. Error Model and Tracking Control Problems}

In this paper, we consider the following unicycle-modeled mobile robots [27]

$$
\begin{aligned}
\dot{x} & =v \cos \theta \\
\dot{y} & =v \sin \theta \\
\dot{\theta} & =w
\end{aligned}
$$

where $(x, y)$ are the Cartesian coordinates, $\theta$ is the angle between the heading direction of the mobile robot and the $x$ axis, $v$ is the linear velocity, and $w$ the angular velocity. Note that a mobile robot on a plane (1) possesses three degrees of freedom of motion which must be controlled by only two control inputs and under nonholonomic constraint. Many researchers have shown that according to Brockett's theorem [3], such a system is open-loop controllable, but not stabilizable, by pure smooth time-invariant feedback [30].

Suppose a reference trajectory $\left(x_{r}(t), y_{r}(t), \theta_{r}(t)\right)$ is described by

$$
\begin{aligned}
\dot{x}_{r} & =v_{r} \cos \theta_{r} \\
\dot{y}_{r} & =v_{r} \sin \theta_{r} \\
\dot{\theta}_{r} & =w_{r} .
\end{aligned}
$$

Throughout this paper, we assume that $v_{r}(t)$ and $w_{r}(t)$ are both piecewise continuous and bounded functions. The following is a tracking control problem for (1).

Tracking Control Problem (TCP): To find control laws for $v$ and $w$ such that (1) follows a reference trajectory $\left(x_{r}(t), y_{r}(t), \theta_{r}(t)\right)$. That is, $\lim _{t \rightarrow \infty}\left|x(t)-x_{r}(t)\right|=0$, $\lim _{t \rightarrow \infty}\left|y(t)-y_{r}(t)\right|=0$, and $\lim _{t \rightarrow \infty}^{t \rightarrow \infty}\left|\theta(t)-\theta_{r}(t)\right|=0$.

In practical applications, the following practical tracking problem can be more easily handled. It achieves a convergence effect similar to that stated in the TCP.

Practical Tracking Control Problem (PTCP): For any desired tracking error bound $\varepsilon>0$, find control laws for $v$ and $w$ such that there exists a positive constant, $T_{0}$ satisfying the inequality $\mid\left(x(t)-x_{r}(t), y(t)-y_{r}(t), \theta(t)-\right.$ $\left.\theta_{r}(t)\right)^{T} \mid \leq \varepsilon, \forall t \geq T_{0}$. In other words, (1) follows a reference trajectory $\left(x_{r}(t), y_{r}(t), \theta_{r}(t)\right)$ up to the given tracking error $\varepsilon$.

For convenience, we choose the new coordinates and inputs [21]:

$$
\begin{aligned}
& {\left[\begin{array}{l}
x_{0} \\
x_{1} \\
x_{2}
\end{array}\right]=\left[\begin{array}{ccc}
0 & 0 & 1 \\
-\sin \theta & \cos \theta & 0 \\
-\cos \theta & -\sin \theta & 0
\end{array}\right]\left[\begin{array}{l}
x_{r}-x \\
y_{r}-y \\
\theta_{r}-\theta
\end{array}\right]} \\
& {\left[\begin{array}{l}
u_{0} \\
u_{1}
\end{array}\right]=\left[\begin{array}{c}
w_{r}-w \\
v-v_{r} \cos x_{0}
\end{array}\right] .}
\end{aligned}
$$

The tracking error model is then obtained as

$$
\begin{aligned}
& \dot{x}_{0}=u_{0} \\
& \dot{x}_{1}=\left(w_{r}-u_{0}\right) x_{2}+v_{r} \sin x_{0} \\
& \dot{x}_{2}=-\left(w_{r}-u_{0}\right) x_{1}+u_{1} .
\end{aligned}
$$

System (4a)-(4c) is referred to as the error model of the TCP and PTCP. The coordinates $\left(x_{0}, x_{1}, x_{2}\right)^{T}$ are used throughout this paper in solving tracking problems. Since the coordinate transformation (3) is an orthogonal transformation, we have

$$
\left|\left(x_{0}, x_{1}, x_{2}\right)^{T}\right|=\left|\left(x-x_{r}, y-y_{r}, \theta-\theta_{r}\right)^{T}\right| .
$$

In particular, $\left(x_{0}, x_{1}, x_{2}\right)=0$ is equivalent to $x=x_{r}, y=y_{r}$, and $\theta=\theta_{r}$. Thus, if $\left(x_{0}, x_{1}, x_{2}\right)$ converges to zero, then the TCP is solved. With the new coordinates $\left(x_{0}, x_{1}, x_{2}\right)$, the TCP 
has now been transformed into a stability problem. The same transformation applies to the PTCP.

\section{B. Transforming Parking Problems Into Tracking Problems via Virtual Trajectory}

In practical applications, a mobile robot must stop at certain place with a prescribed pose. Such problems are called parking problems. In this subsection, we solve the PTCP, rather than the TCP, for the parking problem in which parking means the desired linear and angular velocities that satisfy the following assumption.

(P) (Assumption for Parking Problem): Suppose there is a constant $T_{f} \geq 0$ such that $v_{r}(t)=0$, and $w_{r}(t)=0, \forall t \geq T_{f}$, and also that the limit $\lim _{t \rightarrow T_{f}^{-}} w_{r}(t)=M_{w}$ exists when $T_{f}>0$.

Remark 1: The restriction on the existence of the limit $\lim _{t \rightarrow T_{f}^{-}} w_{r}(t)$ is to make sure that the modified signals are continuous on $[0, \infty)$ whenever the original signal $w_{r}(t)$ is continuous on $\left[0, T_{f}\right)$. This assumption is always true in most practical applications. Because of implementation considerations, it is more preferable to track continuous signals than discontinuous signals.

Our solution to parking problems combines a motion-planning method with a tracking controller design. First, consider the following set of continuous periodic functions defined on $[0, \infty)$.

Definition 1: For any positive constant $M$, let $S_{M}$ be the set of all continuous real-valued periodic functions $h$ defined on $[0, \infty)$ that satisfy the following conditions:

1) $\int_{0}^{T} h(t) d t=0$, where $T$ is the period of $h$.

2) There exist two constants $t_{0} \geq 0$ and $s_{0} \geq 0$ such that $h\left(t_{0}\right)=M$ and $h\left(s_{0}\right)=-M$.

Note that $S_{M}$ contains many functions for any given positive constant $M$, for instance, $M \sin \omega t \in S_{M}, \forall \omega \neq 0$. Below, a virtual trajectory is added to the original trajectory. Let $M_{w}$ be the constant defined in Assumption (P). Consider the set $S_{\bar{M}}$ with $\bar{M} \geq\left|M_{w}\right|$ being a positive constant. Let $w_{f}(t) \in S_{\bar{M}}$. By the mean value theorem [31] and the definition of $S_{\bar{M}}$, there exists a $\bar{t}_{0} \geq 0$ such that $w_{f}\left(\bar{t}_{0}\right)=M_{w}$. The desired angle $\theta_{r}(t)$ and angular velocity $w_{r}(t)$ are modified as follows:

$$
\begin{aligned}
& \tilde{\theta}_{r}(t)=\left\{\begin{array}{ll}
\theta_{r}(t), & \text { if } 0 \leq t<T_{f} \\
\theta_{r}\left(T_{f}\right)+\int_{\bar{t}_{0}}^{t-T_{f}+\bar{t}_{0}} w_{f}(\tau) d \tau, & \text { if } t \geq T_{f}
\end{array}\right. \text { and } \\
& \widetilde{w}_{r}(t)= \begin{cases}w_{r}(t), & \text { if } 0 \leq t<T_{f} \\
w_{f}\left(t-T_{f}+\bar{t}_{0}\right), & \text { if } t \geq T_{f} .\end{cases}
\end{aligned}
$$

Notice that (2) still holds when the functions $\theta_{r}(t)$ and $w_{r}(t)$ are replaced by $\widetilde{\theta}_{r}(t)$ and $\widetilde{w}_{r}(t)$ under Assumption (P). Moreover, as specified in Remark 1, $\widetilde{w}_{r}(t)$ is a continuous function defined on $[0, \infty)$ whenever the original signal $w_{r}(t)$ is continuous on $\left[0, T_{f}\right)$. The following proposition is used in solving the parking problem in the next section.

Proposition 1: Suppose the Assumption (P) holds and there exist controllers $\left(v^{*}, w^{*}\right)$ that solve the TCP when the functions $\theta_{r}(t)$ and $w_{r}(t)$ are replaced by $\widetilde{\theta}_{r}(t)$ and $\widetilde{w}_{r}(t)$. Then, the PTCP for the original reference trajectory is also solvable by the following modified controllers:

$$
(v, w)= \begin{cases}\left(v^{*}, w^{*}\right), & \text { if } 0 \leq t \leq T_{0} \\ (0,0), & \text { if } t>T_{0}\end{cases}
$$

where for any given positive constant (error bound) $\varepsilon$, $T_{0}(\varepsilon) \geq T_{f}$ is any constant satisfying $\mid\left(x\left(T_{0}\right)-x_{r}\left(T_{0}\right), y\left(T_{0}\right)-\right.$ $\left.y_{r}\left(T_{0}\right), \theta\left(T_{0}\right)-\theta_{r}\left(T_{0}\right)\right)^{T} \mid<\varepsilon$.

Proof: Let $T$ be the period of $w_{f}(t)$, that is the function given in (6). Then, $\widetilde{\theta}_{r}\left(T_{f}+k T\right)=\widetilde{\theta}_{r}\left(T_{f}\right)=\theta_{r}\left(T_{f}\right)=\theta_{r}\left(T_{f}+\right.$ $k T), \forall k \in \aleph$, by Assumption (P) and the periodic property of $w_{f}(t)$. Since the controller $\left(v^{*}, w^{*}\right)$ solves the TCP when the functions $\theta_{r}(t)$ and $w_{r}(t)$ are replaced by $\widetilde{\theta}_{r}(t)$ and $\widetilde{w}_{r}(t)$, respectively, we have

$$
\begin{gathered}
\lim _{k \rightarrow \infty} \mid\left(x\left(T_{f}+k T\right)-x_{r}\left(T_{f}+k T\right), y\left(T_{f}+k T\right)\right. \\
\left.-y_{r}\left(T_{f}+k T\right), \theta\left(T_{f}+k T\right)-\theta_{r}\left(T_{f}+k T\right)\right)^{T} \mid \\
=\lim _{k \rightarrow \infty} \mid\left(x\left(T_{f}+k T\right)-x_{r}\left(T_{f}+k T\right)\right. \\
y\left(T_{f}+k T\right)-y_{r}\left(T_{f}+k T\right) \\
\left.\theta\left(T_{f}+k T\right)-\widetilde{\theta}_{r}\left(T_{f}+k T\right)\right)^{T} \mid=0 .
\end{gathered}
$$

In particular, for any given positive constant $\varepsilon$, there exists a constant $T_{0}(\varepsilon) \geq T_{f}$ that satisfies $\mid\left(x\left(T_{0}\right)-x_{r}\left(T_{0}\right), y\left(T_{0}\right)-\right.$ $\left.y_{r}\left(T_{0}\right), \theta\left(T_{0}\right)-\theta_{r}\left(T_{0}\right)\right)^{T} \mid \leq \varepsilon$. Notice that for all $t>T_{0}$, the states and the tracking signals are all constant functions by Assumption $(\mathrm{P})$, and $(v, w)=(0,0)$ in this case. Hence, the modified controllers (7) solve the PTCP. This completes the proof.

Remark 2: Proposition 1 proposes a general framework for solving the parking problem. The proposed method requires an open-loop generator satisfying some PE conditions to transform the parking problem into a tracking problem. Recently, [10] and [26] proposed another powerful tool for solving the PTCP without employing an open-loop generator. However, the benefit of our approach is that various tracking controllers proposed in current literature (in, for example, [11], [15], [20], and [21]) can be adopted to solve the parking problem with the framework described in Proposition 1. Thus, our approach provides more possibilities for choosing parking controllers. See the next section for details.

\section{GlobAl TRACKING CONTROL LAWS WITH APPLICATIONS TO PARKING PROBLEMS}

\section{A. A Modified Tracking Controller}

In this section, the TCP is solved when the tracking trajectory is moving, the parking problem is also solved using a virtual moving trajectory according to Proposition 1. Exponential convergence is guaranteed for the proposed controllers, thus improving convergence rates.

Let $\alpha(0)=1$ and $\alpha\left(x_{0}\right)=\left(\sin x_{0} / x_{0}\right)$ if $x_{0} \neq 0 . \alpha\left(x_{0}\right)$ is then a smooth function and $\sin \left(x_{0}\right)=x_{0} \alpha\left(x_{0}\right)$. Choose a Lyapunov function

$$
V=\frac{1}{2}\left(a_{0} x_{0}^{2}+a_{1} x_{1}^{2}+a_{2} x_{2}^{2}\right)
$$


where $a_{0}, a_{1}$, and $a_{2}$ are three positive constants. In view of Lyapunov theory [37], controllers can be given as follows:

$$
\begin{aligned}
& \left(u_{0}, u_{1}\right)=\left(-\left(\frac{a_{1}}{a_{0}}\right) v_{r} \alpha\left(x_{0}\right) x_{1}-k_{0} x_{0}\right. \\
& \left.\left(1-\frac{a_{1}}{a_{2}}\right)\left(w_{r}-u_{0}\right) x_{1}-\left(\left|w_{r}-u_{0}\right|+k_{2}(t)\right) a_{2} x_{2}\right)
\end{aligned}
$$

where $k_{0}$ is any positive constant and $k_{2}(t)$ is a piecewise continuous and bounded nonnegative function. We then have

$$
\dot{V}=-\left(a_{0} k_{0} x_{0}^{2}+\left(\left|w_{r}-u_{0}\right|+k_{2}(t)\right) a_{2}^{2} x_{2}^{2}\right) \leq 0
$$

using direct computation.

Consider the following conditions. They prescribe that the desired trajectory is a moving trajectory and can also be viewed as persistent excitation conditions [21], [28].

C1) There exist three positive constants $T_{0}, r$, and $\delta$ such that

$$
\left|\int_{s_{0}}^{s_{0}+\delta} v_{r}(\tau) d \tau\right|+\left|\int_{s_{0}}^{s_{0}+\delta} w_{r}(\tau) d \tau\right|>r
$$

for all $t \geq 0$ and some $t \leq s_{0} \leq t+T_{0}$.

C2) There exist three positive constants $T_{0}, r$, and $\delta$ such that

$$
\left|\int_{s_{0}}^{s_{0}+\delta} w_{r}(\tau) d \tau\right|>r
$$

for all $t \geq 0$ and some $t \leq s_{0} \leq t+T_{0}$.

Obviously, Condition C2) implies Condition C1). These conditions can be employed in various situations. The following theorem, based on Conditions $\mathrm{C} 1$ ) or $\mathrm{C} 2$ ), can be proposed. Its proof is given in the Appendix.

Theorem 1: Consider the system (4a)-(4c) with controllers chosen per (9). Then, the origin is uniformly globally asymptotically stable and locally exponentially stable under Condition C2). In addition to $k_{2}(t) \geq k_{1}>0, \forall t \geq 0$, the same result holds under the weaker Condition $\mathrm{C} 1$ ).

\section{B. Improving Parking Performance: Linearization and Pole Placement}

Theorem 1 only guarantees locally exponential stability. The exact convergence rate is still unknown. In this subsection, a further analysis of performance is presented employing a kind of linearization called time-scaling [32]. In particular, a poleplacement method is used here.

Since the main concern in this paper is the parking problem, we assume the desired linear velocity to be $v_{r}(t) \equiv 0$. In this case, Conditions $\mathrm{C} 1$ ) and $\mathrm{C} 2$ ) are equivalent. Moreover, the time function $k_{2}(t)$ is set to $k_{2} \equiv 0$. The following lemma is used to estimate the convergence rate of the closed-loop system.

Lemma 1: Suppose Condition C2) holds. Let $\tau(t):[0, \infty) \times$ $\Re \rightarrow[0, \infty)$ be defined as

$$
\tau\left(t, x_{0}\right)=\int_{0}^{t}\left|w_{r}(s)+k_{0} x_{0} e^{-k_{0} s}\right| d s, \quad \forall t \geq 0, \quad \forall x_{0} \in \Re
$$

for some positive constant $k_{0}$. The following inequality then holds:

$$
\tau\left(t+T_{0}+\delta, x_{0}\right)-\tau\left(t, x_{0}\right) \geq r-\left|x_{0}\right| e^{-k_{0} t}, \quad \forall t \geq 0 .
$$

In particular, for each $x_{0}$ in $\Re$, we have $\lim \tau\left(t, x_{0}\right)=\infty$.

Proof: In view of Condition C2), $\left(\begin{array}{l}t \rightarrow \infty \\ (14)\end{array}\right.$ inequality

$$
\begin{aligned}
\tau(t & \left.+T_{0}+\delta, x_{0}\right)-\tau\left(t, x_{0}\right) \\
& =\int_{t}^{t+T_{0}+\delta}\left|w_{r}(s)+k_{0} x_{0} e^{-k_{0} s}\right| d s \\
& \geq \int_{s_{0}}^{s_{0}+\delta}\left|w_{r}(s)\right| d s-\int_{s_{0}}^{s_{0}+\delta} k_{0}\left|x_{0}\right| e^{-k_{0} s} d s \\
& \geq r-\left|x_{0}\right| e^{-k_{0} t}
\end{aligned}
$$

for all $t \geq 0$, all $x_{0} \in \Re$, and some $t \leq s_{0} \leq t+T_{0}$. This completes the proof.

By the assumption $v_{r}(t) \equiv 0$ and setting the controllers (9) to $k_{2} \equiv 0$, the closed-loop system (4a)-(4c) can be written in the following form:

$$
\begin{aligned}
& \dot{x}_{0}=-k_{0} x_{0} \\
& \dot{x}_{1}=\left(w_{r}+k_{0} x_{0}\right) x_{2} \\
& \dot{x}_{2}=-\left(\frac{a_{1}}{a_{2}}\right)\left(w_{r}+k_{0} x_{0}\right) x_{1}-a_{2}\left|w_{r}+k_{0} x_{0}\right| x_{2} .
\end{aligned}
$$

Consider first the following augmented second-order linear systems:

$$
\left[\begin{array}{l}
\dot{\bar{x}}_{1} \\
\overline{\bar{x}}_{2}
\end{array}\right]=\left[\begin{array}{cc}
0 & \sigma \\
-\sigma \frac{a_{1}}{a_{2}} & -a_{2}
\end{array}\right]\left[\begin{array}{l}
\bar{x}_{1} \\
\bar{x}_{2}
\end{array}\right]
$$

where $\sigma= \pm 1$. It is easy to see that the characteristic values $\lambda_{1}$, $\lambda_{2}$ of system matrices for $\sigma= \pm 1$ are the same. Moreover, it can be verified that

$$
\lambda_{1}+\lambda_{2}=-a_{2} \quad \text { and } \quad \lambda_{1} \lambda_{2}=\frac{a_{1}}{a_{2}} .
$$

Below, we show that (16) is a kind of linearization of the nonlinear system (15b), (15c) by using the time-scaling method. Let the function $\operatorname{sign}(x)$ be the sign function, i.e., $\operatorname{sign}(x)=1$ if $x>0$, and $\operatorname{sign}(x)=-1$ if $x<0$. Define the time-scaling function $\tau\left(t, x_{0}(0)\right)$ as in (13). Suppose the sign of $w_{r}(t)+$ $k_{0} x_{0}(0) e^{-k_{0} t}$ is constant for some time interval $(a, b)$. The solution $\left(x_{1}(t), x_{2}(t)\right)^{T}$ of $(15 \mathrm{~b}),(15 \mathrm{c})$ can then be expressed as

$$
\begin{aligned}
\left(x_{1}(t), x_{2}(t)\right)^{T}=\left(\bar{x}_{1}\left(\tau\left(t, x_{0}(0)\right)\right)\right. & \\
& \left.\bar{x}_{2}\left(\tau\left(t, x_{0}(0)\right)\right)\right)^{T} \quad \forall t \in[a, b]
\end{aligned}
$$

where $\left(\bar{x}_{1}(s), \bar{x}_{2}(s)\right)^{T}$ is the solution of (16) on $\left[\tau\left(a, x_{0}(0)\right), \tau\left(b, x_{0}(0)\right)\right] \quad$ with $\sigma=\operatorname{sign}\left(w_{r}(t)+\right.$ $\left.k_{0} x_{0}(0) e^{-k_{0} t}\right)$. Thus, (16) can be used to investigate the behavior of the nonlinear system (15a)-(15c). In particular, we give the following theorem. Please refer to [18] for the proof.

Theorem 2: Let $\lambda_{1}$ and $\lambda_{2}$ be two distinct negative constants. Consider the nonlinear system (15a)-(15c) in which the constants $a_{1}$ and $a_{2}$ are determined by (17). Suppose the sign of $w_{r}(t)+k_{0} x_{0}(0) e^{-k_{0} t}$ is constant for some time interval $(a, b)$. 
Then, every solution $x(t)=\left(x_{1}(t), x_{2}(t)\right)^{T}$ of (15a)-(15c) satisfies the following inequalities:

$$
\begin{aligned}
& |x(t)| \\
& \leq \| V \cdot\left[\begin{array}{lc}
e^{\lambda_{1}\left(\tau\left(t, x_{0}(0)\right)-\tau\left(a, x_{0}(0)\right)\right)} & 0 \\
0 & e^{\lambda_{2}\left(\tau\left(t, x_{0}(0)\right)-\tau\left(a, x_{0}(0)\right)\right)}
\end{array}\right] \\
& \cdot V^{-1}|||x(a)|, \quad \forall a \leq t \leq b
\end{aligned}
$$

where $V=\left[\begin{array}{cc}1 & 1 \\ \lambda_{1} & \lambda_{2}\end{array}\right]$ is the Vandermode matrix and $\tau\left(t, x_{0}(0)\right)$ is the time-scaling function defined in (13). $|x(t)| \leq M_{0}|x(a)|, \forall a \leq t \leq b$, with

$$
M_{0}=\max _{s \geq 0}\left\|V \cdot\left[\begin{array}{cc}
e^{\lambda_{1} s} & 0 \\
0 & e^{\lambda_{2} s}
\end{array}\right] V^{-1}\right\| \geq 1 .
$$

Remark 3: Theorem 2 gives estimates for the effect of pole placement. For example, the convergence rate can be computed using inequalities (14) and (19), and transient behavior (maximum overshoot) can be estimated using inequality (20). Numerical methods can also be employed to minimize $M_{0}$, thus improving transient behavior. Furthermore, observations concerning the robustness of the closed-loop system (15) can also be made, but since space is limited here and they do not affect our topic, we omit the details and refer readers to [18].

\section{Fast Parking Control by Employing Tracking Controllers}

In this subsection, we present a solution to the parking problem based on the tracking controller design given in preceding subsections; we assume Assumption (P) holds throughout this subsection. Given Proposition 1, we need only solve the TCP for the modified trajectory. Condition C2) from Theorem 1 remains to be verified. To this end, let $w_{f}(t) \in S_{M}$ be the function in (6) for some positive constant $M$, and $T$ be the period. Then, by the definition of $S_{M}$, there exists a $t_{0} \geq 0$ such that $w_{f}\left(t_{0}\right)=M$. Since $w_{f}(t)$ is continuous and periodic, there exists a positive constant $\delta$ such that $w_{f}(t) \geq w_{f}(s)-M / 2, \forall s \leq t \leq s+\delta$. Let $T_{0}=T_{f}+T$. By the periodic property of $w_{f}(t)$, for any $t \geq 0$, there exists a $t+T_{f} \leq s_{0} \leq t+T_{f}+T=t+T_{0}$ such that $w_{f}\left(s_{0}-T_{f}+\bar{t}_{0}\right)=$ $w_{f}\left(t_{0}\right)=M$, where $\bar{t}_{0}$ is the constant given in (6). This implies that $w_{f}\left(t-T_{f}+\bar{t}_{0}\right) \geq w_{f}\left(s_{0}-T_{f}+\bar{t}_{0}\right)-M / 2=M / 2$, $\forall s_{0} \leq t \leq s_{0}+\delta$. Thus, the following inequality holds:

$\left|\int_{s_{0}}^{s_{0}+\delta} \widetilde{w}_{r}(\tau) d \tau\right|=\left|\int_{s_{0}}^{s_{0}+\delta} w_{f}\left(\tau-T_{f}+\bar{t}_{0}\right) d \tau\right| \geq \frac{M \delta}{2}>\frac{M \delta}{3}$.

In particular, the Condition C2) holds for the modified desired angular velocity $\widetilde{w}_{r}$ defined in (6) with $r=M \delta / 3$. In view of Theorem 2, it is convenient to set $k_{2}(t)=0, \forall t \geq T_{f}$, when applying the pole-placement method. Theorem 3 then follows from Proposition 1, Theorem 1 and (17).

Theorem 3: Let $\lambda_{1}$ and $\lambda_{2}$ be two distinct negative constants, $k_{0}$ and $a_{0}$ be any positive constants, and $k_{2}(t)$ be a piecewise continuous and bounded nonnegative function with $k_{2}(t)=0$, $\forall t \geq T_{f}$. Suppose Assumption (P) holds. Replace the modified trajectory $\theta_{r}(t)$ and $w_{r}(t)$ with the functions $\widetilde{\theta}_{r}(t)$ and $\widetilde{w}_{r}(t)$ defined in (6), and the PTCP is then solvable by the following controllers:

$$
(v, w)= \begin{cases}\left(v^{*}, w^{*}\right), & \text { if } 0 \leq t \leq T_{0} \\ (0,0), & \text { if } t>T_{0}\end{cases}
$$

where for any given positive constant $\varepsilon, T_{0}(\varepsilon) \geq T_{f}$ is any constant satisfying $\mid\left(x\left(T_{0}\right)-x_{r}\left(T_{0}\right), y\left(T_{0}\right)-y_{r}\left(T_{0}\right), \theta\left(T_{0}\right)-\right.$ $\left.\theta_{r}\left(T_{0}\right)\right)^{T} \mid<\varepsilon$, and $w^{*}$ and $v^{*}$ are as described in (22), shown at the bottom of the page.

Remark 4: The function $k_{2}(t)$ is a tuning function. In most applications, tracking signals satisfy either Condition C1) or Assumption (P). Roughly speaking, this situation can be referred to as being in tracking mode when Condition $\mathrm{C} 1$ ) holds and being in parking mode when Assumption (P) holds. Given Theorem 1, it is preferable to set the tuning function $k_{2}(t)$ to a nonzero value in tracking mode, but according to Theorem 2, it is preferable to set the tuning function $k_{2}(t)$ equal to zero in parking mode in order to use the pole-placement method to improve parking performance. The controller proposed here solves the tracking and parking problems at the same time according to Theorem 1 and Theorem 3 by choosing suitable tuning functions $k_{2}(t)$ and adding virtual tracking signals to the original tracking trajectory. Although a single controller was proposed in [11] and [21] to solve a similar problem, it was found that the convergence rate was very slow in parking mode. However, the controller proposed here can achieve exponential convergence on the parking problem via Theorem 1 . This is demonstrated by means of an interesting experiment in the next section. It was also observed that the controllers presented in the papers mentioned above cannot be extended to nonholonomic systems with orders higher than three due to their highly nonlinear structures. By contrast, the method proposed in this paper can be easily generalized to higher dimension nonholonomic systems. In fact, a successful application of the idea presented in this paper to the parking control of an underactuated ship was recently given in [19].

\section{Simulations AND EXPERIMENTAL RESULTS}

Fig. 1 shows the experimental mobile robot developed in the Intelligent System Control Integration Lab at National Chiao Tung University, Taiwan, R.O.C. It has two independent dc-servo-driven wheels and two casters for balance. Motion is controlled by adjusting the velocities of the left and right wheels via a two-axis motion control card based on a TMS320F240

$$
\left[\begin{array}{c}
w^{*} \\
v^{*}
\end{array}\right]=\left[\begin{array}{c}
\widetilde{w}_{r}-\frac{\lambda_{1} \lambda_{2}\left(\lambda_{1}+\lambda_{2}\right) v_{r} \alpha\left(x_{0}\right) x_{1}}{a_{0}}+k_{0} x_{0} \\
v_{r} \cos x_{0}+\left(1-\lambda_{1} \lambda_{2}\right) w^{*} x_{1}+\left(\lambda_{1}+\lambda_{2}\right)\left(\left|w^{*}\right|+k_{2}(t)\right) x_{2}
\end{array}\right] .
$$




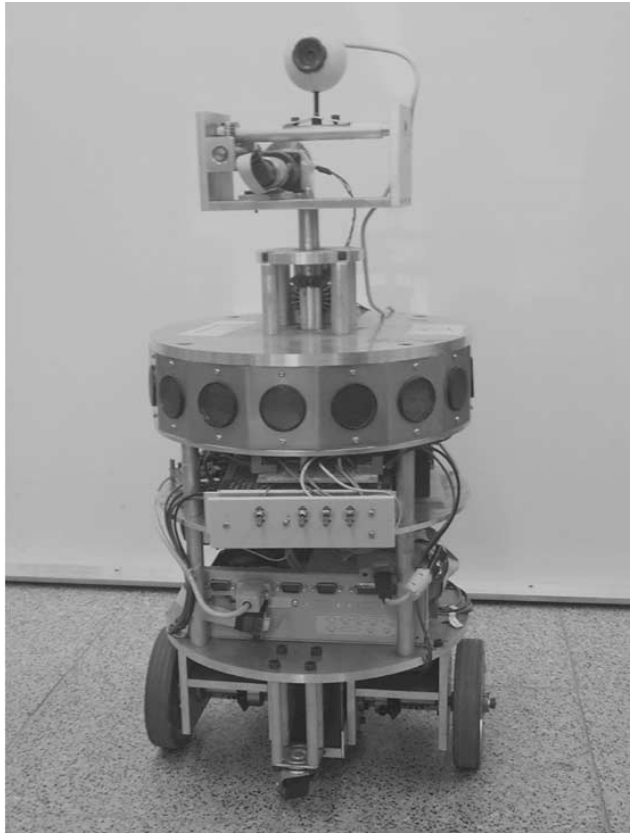

Fig. 1. Experimental mobile robot developed in the Intelligent System Control Integration (ISCI) laboratory at National Chiao Tung University.

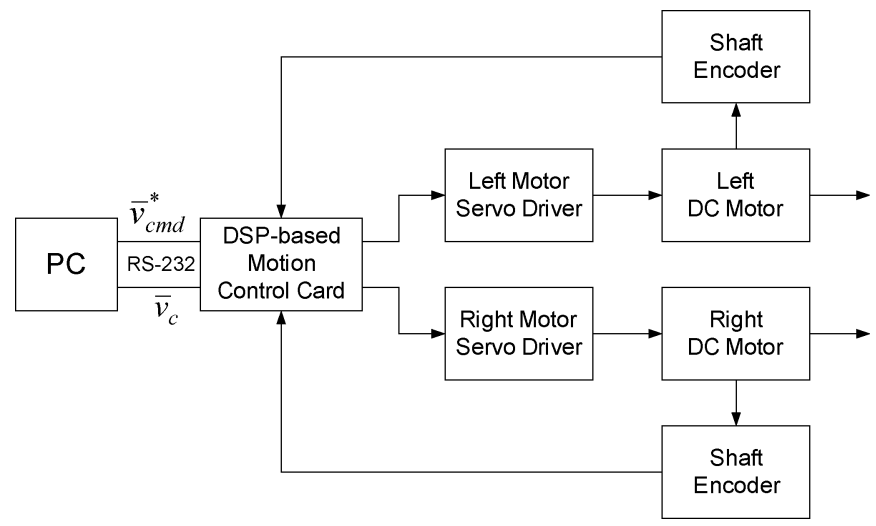

Fig. 2. Block diagram of the wheel motor control system; $\bar{v}_{c m d}^{*}=$ $\left[\begin{array}{ll}v_{l c m d}^{*} & v_{r c m d}^{*}\end{array}\right]^{T}$ is the velocity command vector, and $\bar{v}_{c}=\left[\begin{array}{ll}v_{c} & w_{c}\end{array}\right]^{T}$ is the velocity vector of the robot.

DSP chip from Texas Instruments (TI). Fig. 2 depicts the block diagram of the wheel motor control system. In the figure, $\bar{v}_{c m d}^{*}=\left[\begin{array}{ll}v_{l c m d}^{*} & v_{r c m d}^{*}\end{array}\right]^{T}$ is the velocity command vector, where $v_{l c m d}^{*}$ and $v_{r c m d}^{*}$ denote the velocity commands to the left and right wheels, respectively. $\bar{v}_{c}=\left[\begin{array}{ll}v_{c} & w_{c}\end{array}\right]^{T}$ is the velocity vector of the robot, where $v_{c}$ and $w_{c}$ denote, respectively, the current linear and angular velocities of the robot. The onboard industrial PC only needs to send the velocity commands to the DSP chip, which manages the velocity servo control. Communication between the PC and the motion control card is through an RS-232 serial port.

The proposed parking controller was first verified by computer simulation and then verified by experiments with the mobile robot. The algorithm for estimating robot position is implemented on the PC, which samples the left and right wheel velocities to calculate the current pose of the robot. The velocity of each wheel $v_{l}, v_{r}$ is measured based on the signals from the shaft encoders mounted on the motors. The formula for this pose estimator is

$$
\begin{aligned}
v_{c} & =\frac{v_{l}+v_{r}}{2}, \quad w_{c}=\frac{v_{r}-v_{l}}{E} \\
x_{\text {new }} & =x_{\text {old }}+T \cdot v_{c} \cdot \cos \left(\theta_{\text {old }}\right) \\
y_{\text {new }} & =y_{\text {old }}+T \cdot v_{c} \cdot \sin \left(\theta_{\text {old }}\right) \\
\theta_{\text {new }} & =\theta_{\text {old }}+T \cdot w_{c}
\end{aligned}
$$

where $E$ is the distance between two drive wheels, $v_{c}$ and $w_{c}$ are, respectively, the current linear and angular velocities of the robot, and $T$ denotes the sampling period of the PC. In this case, $E=0.3 \mathrm{~m}$ and $T=0.051 \mathrm{~s}$.

The architecture for realization of the proposed parking controller is presented in Fig. 3. The functions of the blocks shown in Fig. 3 are listed below:

1) coordinate transformation: transform coordinates using (3);

2) tracking control law: compute desired velocities using (22);

$3)$ trajectory generator: generate desired tracking trajectories, e.g., (26a)-(26e);

4) virtual trajectory generator: redesign trajectories using (6); and

5) tracking error checking: compute tracking errors

$$
\left|\bar{s}_{e}\right|=\left|\left(x(t)-x_{r}(t), y(t)-y_{r}(t), \theta(t)-\theta_{r}(t)\right)^{T}\right| .
$$

If $t \geq T_{f}$ and $\left|\bar{s}_{e}\right|<\varepsilon$, stop executing the tracking control law; otherwise, keep tracking.

$\varepsilon$ and $T_{f}$ are, respectively, the desired error bound and finish time for the desired trajectory tracking. They are given in the task specification. The desired velocities $v$ and $w$, calculated by the tracking controller, are transformed into left- and rightwheel velocity commands using

$$
v_{l c m d}^{*}=v-\frac{E \cdot w}{2}, \quad v_{r c m d}^{*}=v+\frac{E \cdot w}{2} .
$$

Fig. 4 shows the complete parking control system constructed using the proposed algorithm. The functions of the blocks shown in Fig. 4 are listed below:

1) DSP-based motion control card: estimate robot velocities using (23);

2) pose estimator: estimate robot positions using (24); and

3 ) velocity transformation: compute velocity commands using (25).

Two trajectories, parallel-parking and back-into-garage, were selected to verify the performance of the proposed parking controller. Fig. 5(a) illustrates the parking place and the parallel parking path, where $2 a$ and $b$ represent, respectively, the long and short axes. In the simulation and experiment, the constants $a$ and $b$ were set to $a=0.4 \mathrm{~m}$ and $b=0.4 \mathrm{~m}$. The trajectory is given in (26a)-(26e). Fig. 5(b) shows the parking place and the back-into-garage path, where $L_{x}, L_{y}$ are the horizontal and vertical distances between the initial and target positions, and $v_{s}, w_{s}$ are the desired linear and angular velocities. In the experiment, these constants were set to $L_{x}=L_{y}=1 \mathrm{~m}$, 


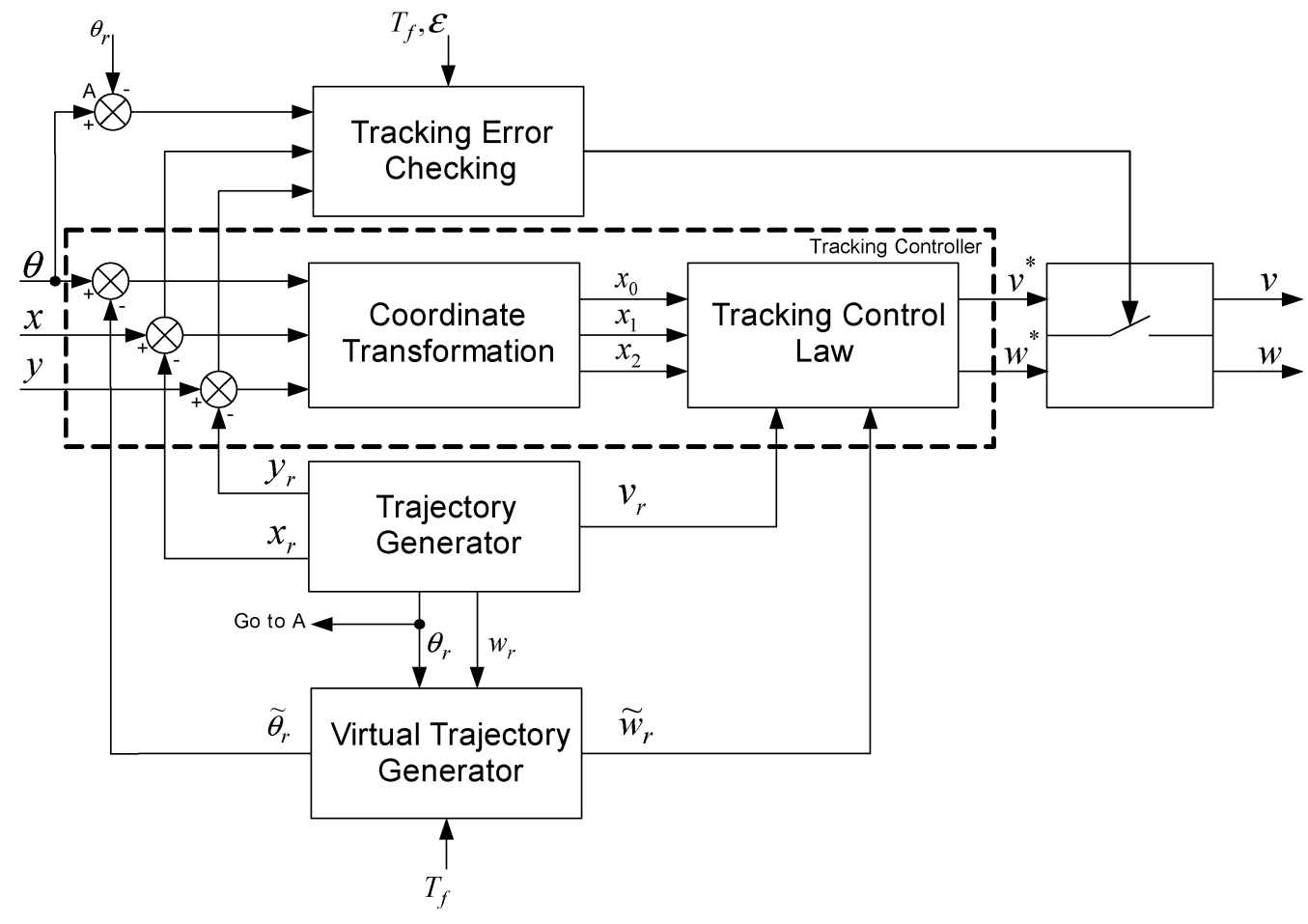

Fig. 3. Developed parking controller.

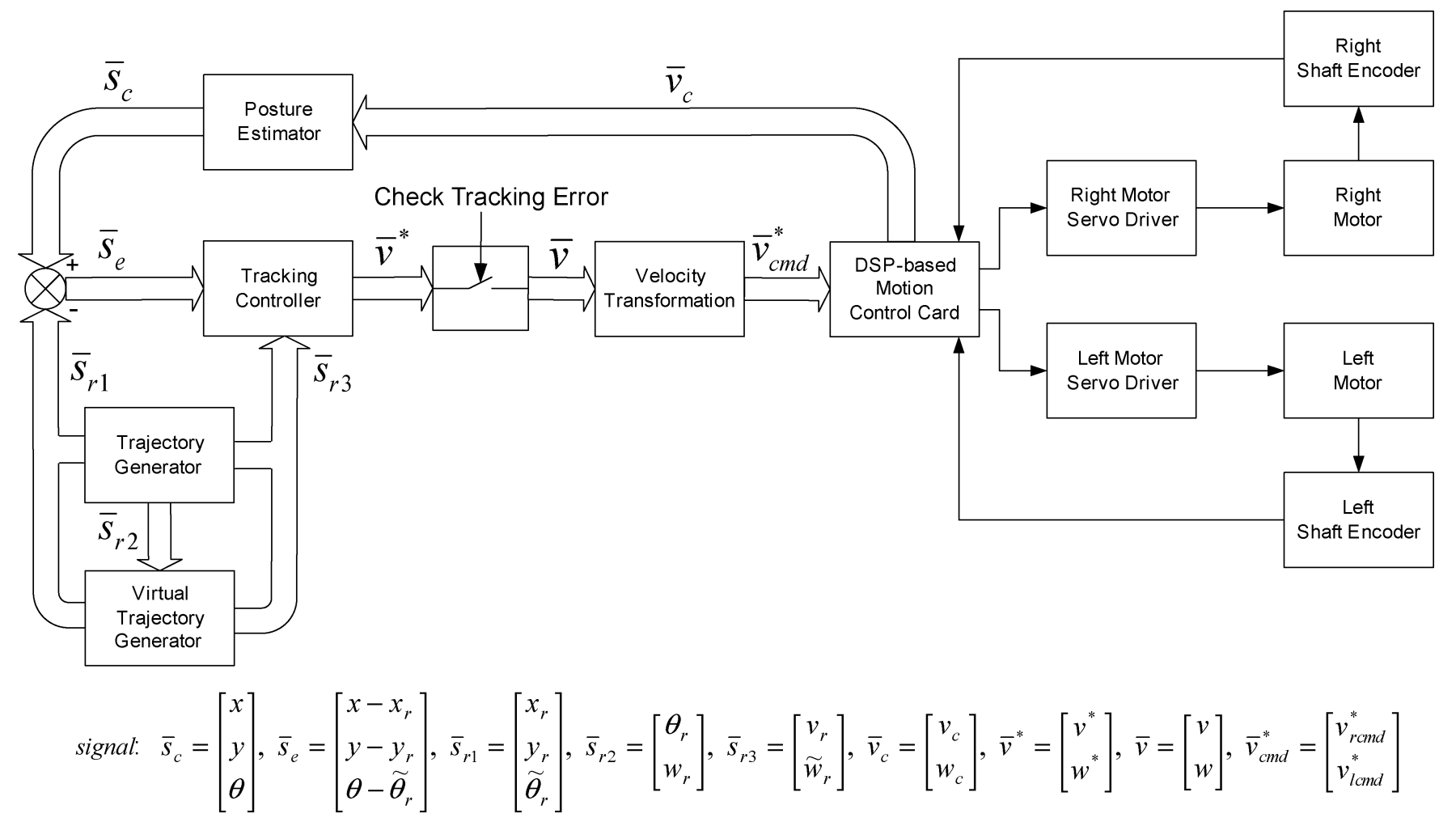

Fig. 4. Implemented parking control system.

$v_{s}=0.0501 \mathrm{~m} / \mathrm{s}$ and $w_{s}=0.5 \mathrm{rad} / \mathrm{s}$. The trajectory equations are given in (28a)-(28e).

In practical applications, a desired error bound $\varepsilon$ must be specified. One way to determine this value is to consider tracking errors in individual axes. We set the desired tracking error to $\left[\begin{array}{lll}0.035 & 0.035 & 0.1\end{array}\right]^{T}$ for each axis, thus $\varepsilon$ is 0.1117 $\left(>\left|\left[\begin{array}{lll}0.035 & 0.035 & 0.1\end{array}\right]^{T}\right|\right)$. As soon as the tracking error $\left|\bar{s}_{e}\right|$ drops below $\varepsilon$ (see Fig. 6 ) for some $t \geq T_{f}$, the controller is set to zero and then the robot stops. The parameters $\left(a_{0}, k_{0}\right)$ are used to match the attainable velocity of the mobile robot. In the experiment, $a_{0}$ was always equal to 1 and a small value was selected for $k_{0}$. Experience gained in computer simulations 
(a)

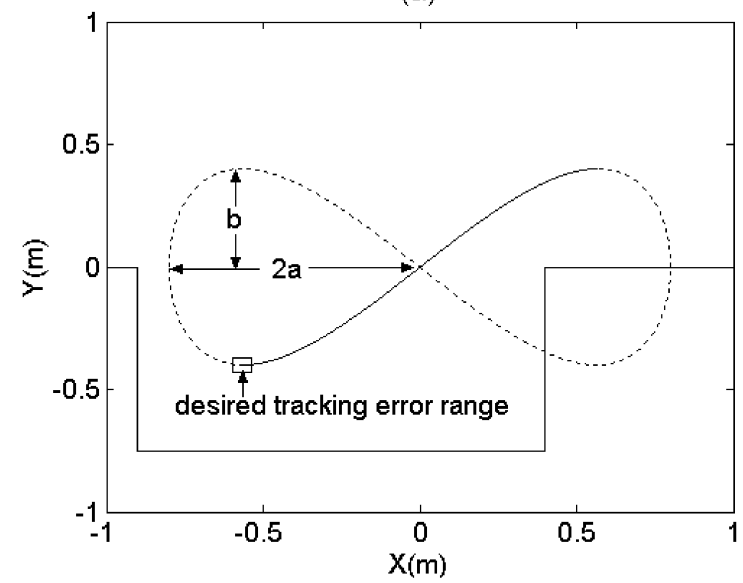

(b)

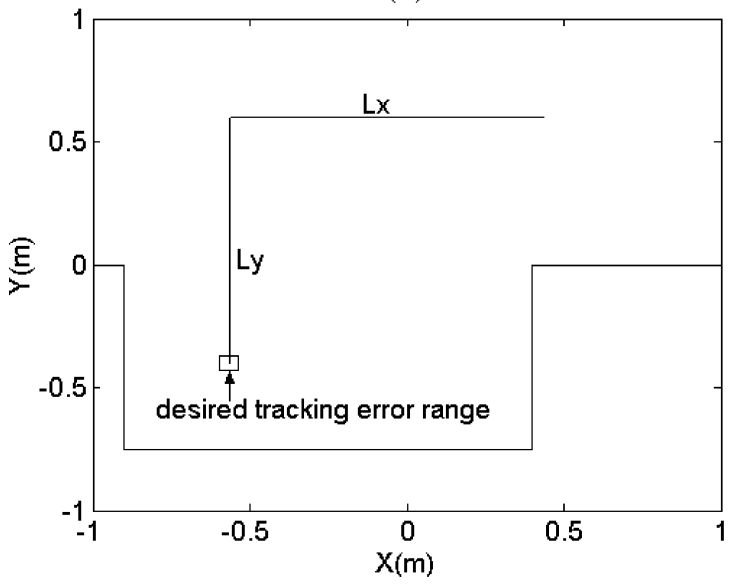

Fig. 5. Parking tasks and designed trajectories for the experiments.

suggested setting $k_{0}$ near 0.1 . The nonnegative continuous function $k_{2}(t)$ was designed according to Theorem 1 and Theorem 3. The parameters $\left(\lambda_{1}, \lambda_{2}\right)$ were determined using Theorem 2, and a numerical method was applied to achieve acceptable transient behavior and convergence rate. Here, we selected various locally optimal parameters $\left(\lambda_{1}, \lambda_{2}\right)$ for each experiment. It would be interesting to find globally optimal parameters for the parking controller and optimize performance with $k_{2}(t)$. This may be a direction for future study.

After resolving parameters, $\left(\lambda_{1}, \lambda_{2}\right)$, we verified the tracking and docking performance of the parking controller using parallel-parking and back-into-garage trajectories. The parallel-parking experiment demonstrates the transient behavior and parking performance of the proposed parking controller, and the back-into-garage experiment verifies the effect the nonnegative continuous function $k_{2}(t)$ has on tracking performance.

\section{A. Parallel-Parking Experiment}

We utilized an 8-shaped trajectory [21] in this experiment. Let $T_{f}=\pi / 2 c$ with $c=0.02$. The model that generated the trajectories is delineated below.

- Parallel-parking path (see (26a)-(26e), shown at the bottom of the next page).
TABLE I

Parameters Used in the Parallel Parking SIMULATION AND EXPERIMENTS

\begin{tabular}{c|c|c|c}
\hline$a_{0}$ & $k_{0}$ & $\lambda_{1}$ & $\lambda_{2}$ \\
\hline 1 & 0.10 & -2.00 & -1.90 \\
\hline
\end{tabular}

Let $w_{f}(t)=0.1 \sin (0.1 \times t) \in S_{0.1} . \widetilde{\theta}_{r}(t)$ and $\widetilde{w}_{r}(t)$ can then be found according to (6) as follows:

$$
\left\{\begin{array}{l}
\widetilde{\theta}_{r}(t)=\pi+\cos \left(0.1 \times \bar{t}_{0}\right)-\cos \left(0.1\left(t-T_{f}+\bar{t}_{0}\right)\right) \quad \forall t \geq T_{f} \\
\widetilde{w}_{r}(t)=0.1 \sin \left(0.1\left(t-T_{f}+\bar{t}_{0}\right)\right)
\end{array}\right.
$$

where parameter $\bar{t}_{0}=10\left(\pi+\sin ^{-1}\left(10\left|w_{r}\left(T_{f}^{-}\right)\right|\right)\right)$with $w_{r}\left(T_{f}^{-}\right)=\lim _{t \rightarrow T_{f}^{-}} w_{r}(t)=-2 \sqrt{2} c$. Roughly speaking, $w_{r}(t)$ given in the parallel-parking path satisfied Condition C2) for $t \leq T_{f}$, hence, according to Theorem $1, k_{2}(t)$ could be set to zero for the parallel parking experiment. The locally optimal parameters used in this experiment are shown in Table I.

Fig. 6 presents the parallel-parking simulation and experimental results. The dotted lines represent computer simulation results, the solid lines the corresponding experimental ones. In Fig. 6(a), we see that the simulation trajectory converged rapidly to the desired path as expected. The tracking-error simulation results in Fig. 6(b) show that the proposed parking controller achieved locally exponential convergence.

Fig. 6(c) and (d) shows the recorded motion control card outputs, i.e., the robot's linear and angular velocities. The leaders in Fig. 6(c) and (d) indicates, respectively, the finish times of the desired tracking trajectory $T_{f}=\pi / 2 c$. As stated in Theorem 3, the parking controller is in the tracking mode when $t<\pi / 2 c$, and changes to parking mode when $t \geq \pi / 2 c$. When the controller is in parking mode and the tracking error drops below the desired error bound, it shuts down. Therefore, the simulation results in Fig. 6 demonstrate that the proposed controller stayed in the tracking mode even when the tracking error was smaller than the desired error bound until $t=\pi / 2 c$, and $(v, w)=(0,0)$ for all $t \geq \pi / 2 c$, meaning the conditions for stopping the controller were satisfied.

We observe that in Fig. 6(a), although the simulation trajectory rapidly converged to the desired trajectory, the experimental robot trajectory did not. This was caused by a shaft encoder truncation error and a quantization error from the velocity command, $\bar{v}_{c m d}^{*}$. Our robot's dc motors are connected to the wheels via a 19:7:1 reduction gear and shaft encoders are mounted on the motor axes. Encoder resolution is 2000 pulses (counts) per revolution, which yields $2000 \times 19.7=39400$ pulses per wheel revolution. The wheel diameter in our design is $125 \mathrm{~mm}$. Hence, the actual encoder resolution is $39400 /(125 \pi) \cong 100$ pulses/mm of wheel displacement.

The servo control sampling time is $1 \mathrm{~ms}$. Thus, the minimum observable velocity from the encoder output is 1000 pulses/s, which, given the encoder's resolution, is equal to $1000 / 100=$ $10 \mathrm{~mm} / \mathrm{s}$ or $0.01 \mathrm{~m} / \mathrm{s}$. In other words, because of encoder resolution limitations and servo loop sampling time, estimation of each wheel's velocity is truncated to $0.01 \mathrm{~m} / \mathrm{s}$. This is the source of the robot's velocity measurement truncation error. 



Experimental ----- Simulation

Fig. 6. Parallel parking simulation and experimental results. (a) Position variations. (b) Tracking errors. (c) Linear velocity of the center point, $v_{c}$. (d) Angular velocity, $w_{c}$.

The velocity commands $v_{l c m d}^{*}$ and $v_{r c m d}^{*}$ are quantized to $0.01 \mathrm{~m} / \mathrm{s}$ in our design. Note that the quantization error in the velocity command is dependent on the bit resolution of the motion control card, but the truncation error is not. We designed the quantization error to be equal to the truncation error. As the robot converges to the target position, the truncation and quantization errors cause large tracking errors because the velocity command is small around the goal [17]. In Fig. 6(c), the experimental result shows that when the linear velocity was lower than $0.05 \mathrm{~m} / \mathrm{s}$, these errors greatly affected the practical linear velocity. Hence, in practice, the robot cannot converge to the desired trajectory at any time before $t=\pi / 2 c$. In order to verify this, we included these errors in the computer simulation and the results are given in Fig. 7. In Fig. 7(a), we see that the practical results and the simulation ones, including truncation and quantization errors, are quite consistent. Fig. 7(b) shows that the three tracking errors all converged to zero up to the given error bound as expected, even with the system's error effects.

Fig. 7(c) and (d) shows the robot's recorded linear and angular velocities. In the figure, the simulation results show that

$$
\begin{aligned}
& x_{r}(t)= \begin{cases}2 a \cos \left(c\left(t+\frac{\pi}{4 c}\right)\right) & 0 \leq t<T_{f} \\
-a \sqrt{2} & t \geq T_{f}\end{cases} \\
& y_{r}(t)= \begin{cases}b \sin \left(2 c\left(t+\frac{\pi}{4 c}\right)\right) & 0 \leq t<T_{f} \\
-b & t \geq T_{f}\end{cases} \\
& v_{r}(t)= \begin{cases}2 c \sqrt{a^{2} \sin ^{2}\left(c\left(t+\frac{\pi}{4 c}\right)\right)+b^{2} \cos ^{2}\left(2 c\left(t+\frac{\pi}{4 c}\right)\right)} & 0 \leq t<T_{f} \\
0 & t \geq T_{f}\end{cases} \\
& \theta_{r}(t)= \begin{cases}\pi-\tan ^{-1}\left(\frac{b \cos \left(2 c\left(t+\frac{\pi}{4 c}\right)\right)}{a \sin \left(c\left(t+\frac{\pi}{4 c}\right)\right)}\right) & 0 \leq t<T_{f} \\
0 & t \geq T_{f}\end{cases} \\
& w_{r}(t)= \begin{cases}\frac{a b c\left(\cos \left(c\left(t+\frac{\pi}{4 c}\right)\right) \cos \left(2 c\left(t+\frac{\pi}{4 c}\right)\right)+2 \sin \left(c\left(t+\frac{\pi}{4 c}\right)\right) \sin \left(2 c\left(t+\frac{\pi}{4 c}\right)\right)\right)}{\left(a \sin \left(c\left(t+\frac{\pi}{4 c}\right)\right)\right)^{2}+\left(b \cos \left(2 c\left(t+\frac{\pi}{4 c}\right)\right)\right)^{2}} & 0 \leq t<T_{f} \\
0 & t \geq T_{f} .\end{cases}
\end{aligned}
$$



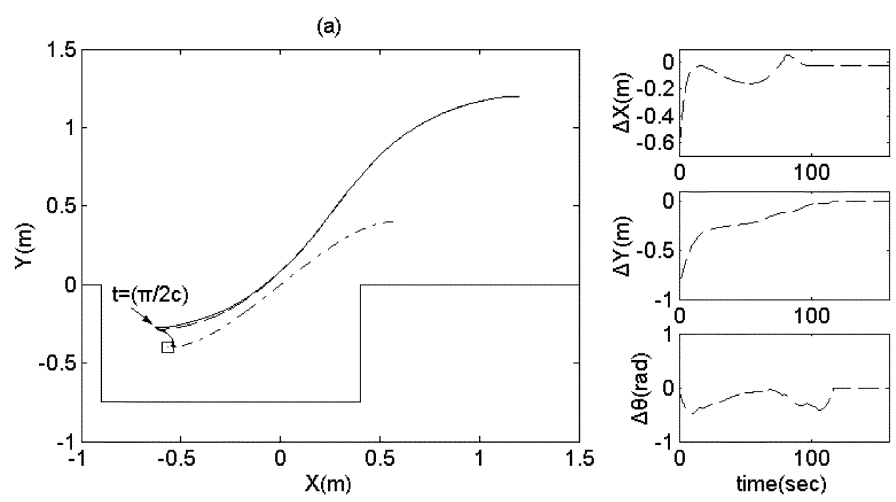

(b)

(c)
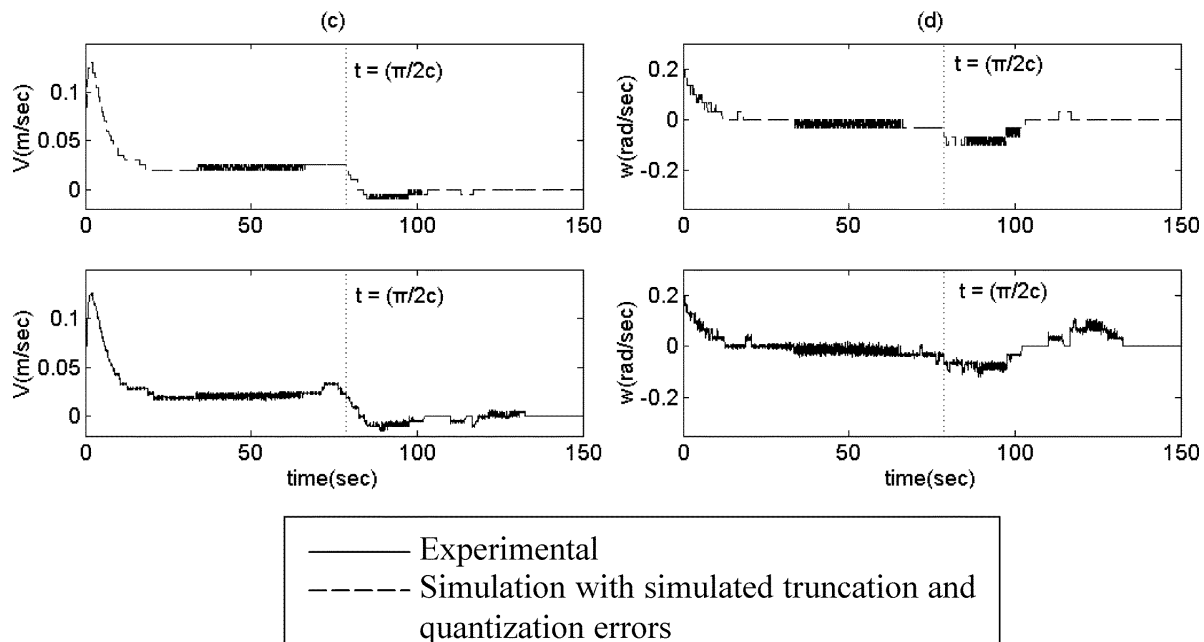

quantization errors

Fig. 7. Experimental results with parallel parking truncation and quantization errors. (a) Position variations. (b) Tracking errors (c) Linear velocity of the center point, $v_{c}$. (d) Angular velocity, $w_{c}$.

the tracking error was larger than the desired error bound at time $t=\pi / 2 c$ and that the controller kept tracking a virtual trajectory until the tracking error was below the desired error bound. Thus, the proposed parking controller overcame this problem although the situation was not considered in the design phase. This is because a virtual trajectory was redesigned for the controller when $t>\pi / 2 c$, and thus transformed the parking problem into a tracking problem. Hence the robot remained in tracking mode until its tracking errors converged to the desired error bound.

In order to emphasize the parking performance of the proposed parking controller, we compared it to the saturation feedback controller proposed in [21]. The experimental results are given in Fig. 8. The dotted lines represent the results from the saturation feedback controller, the solid lines the corresponding results from the proposed parking controller. In Fig. 8(a), we see that the saturation feedback controller trajectory did not converge to the desired path. This shows that the saturation feedback controller cannot solve the parking problem efficiently due to the slow convergence rate. A virtual trajectory was redesigned for the parking controller proposed here to solve the problem when $t>\pi / 2 c$, and the robot remains in tracking mode until its tracking errors converge to the desired error bound. Therefore, combining motion planning with tracking control enhances parking performance such that previous parking control limitations become more amenable to solution.
In Fig. 8(b), we see that when the proposed parking controller's tracking errors all converge to zero, the robot has reached the target position, while the saturation feedback controller still shows tracking errors. Fig. 8(c) and (d) depicts the robots' recorded linear and angular velocities. These simulation and experimental results demonstrate that the proposed parking controller not only solves the general parking problem, but also guarantees rapid convergence rates. This shows that in practice, the parking problem can be solved by combining motion planning with tracking control.

Fig. 9 shows photos of a robot motion sequence during the parallel parking experiment, the black line on the floor indicates the desired trajectory. In Fig. 9(a), the controller is turned on and the robot starts to track the desired trajectory. In Fig. 9(b) and (c), the robot tracks the desired trajectory as it moves toward the target position. In Fig. 9(d) (time $T_{f}$ ), the robot is near the target position, but the tracking error is still larger than the desired tracking error. In Fig. 9(e), the robot is tracking the desired virtual trajectory. In Fig. 9(f), the robot has arrived at the target position and the tracking error is smaller than the desired tracking error, so the parking procedure stops.

\section{B. Back-Into-Garage Parking Experiment}

This experiment verifies the effect of the nonnegative continuous function $k_{2}(t)$ on tracking performance. We utilized an 
(a)

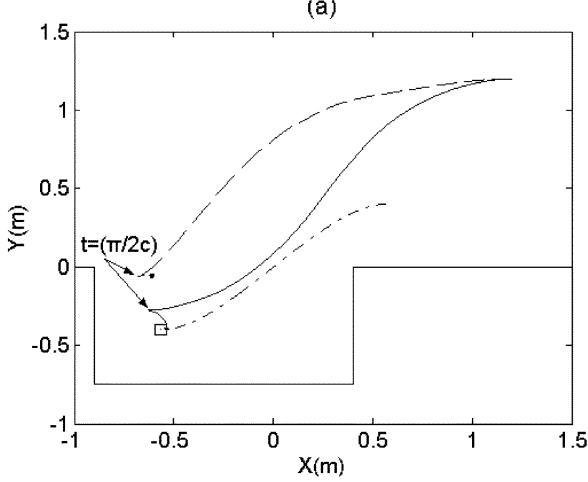

(c)
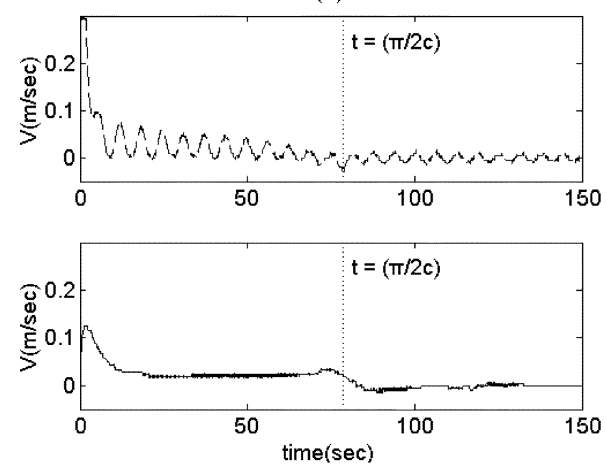

(b)
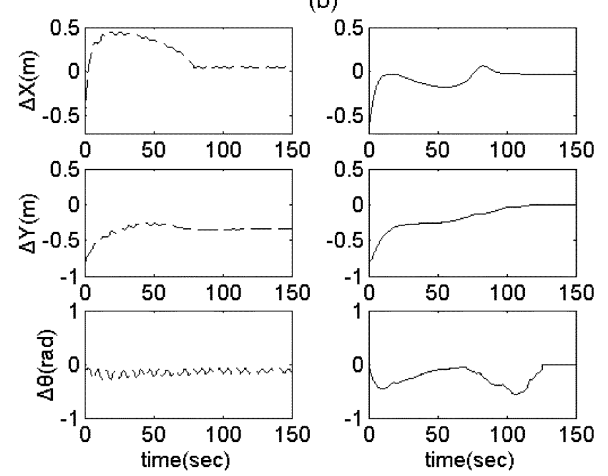

(d)
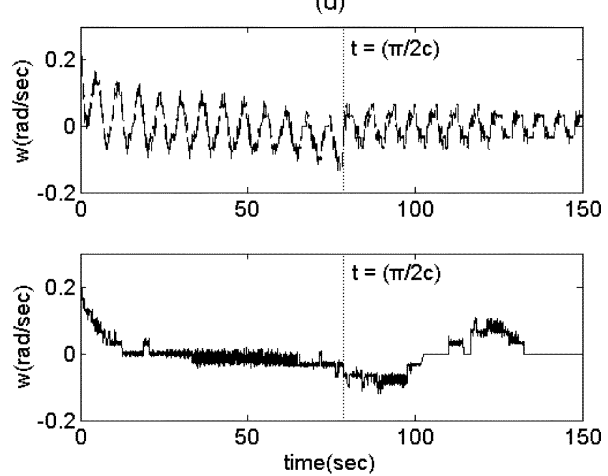

\section{— Proposed Parking Controller} Saturation Feedback Controller

Fig. 8. Comparison of the proposed parking controller and the saturation feedback controller proposed in [21]. (a) Position variations. (b) Tracking errors. (c) Linear velocity of the center point, $v_{c}$. (d) Angular velocity $w_{c}$.

L-shaped trajectory in this experiment. Let $T_{f}=\left(L_{x} / v_{s}\right)+$ $\left(\pi / 2 w_{s}\right)+\left(L_{y} / v_{s}\right)=43 \mathrm{~s}$. The model that generated the trajectories is delineated below

$$
\begin{aligned}
& x_{r}(t)= \begin{cases}x_{s}-v_{s} t, & 0 \leq T<\frac{L_{x}}{v_{s}} \\
x_{f}, & t \geq \frac{L_{x}}{v_{s}}\end{cases} \\
& y_{r}(t)= \begin{cases}y_{s}, & 0 \leq t<\frac{L_{x}}{v_{s}}+\frac{\pi}{2 w_{s}} \\
y_{s}-v_{s}\left(t-\frac{L_{x}}{v_{s}}-\frac{\pi}{2 w_{s}}\right), & \frac{L_{x}}{v_{s}}+\frac{\pi}{2 w_{s}} \leq t<T_{f} \\
y_{f}, & t \geq T_{f}\end{cases} \\
& v_{r}(t)= \begin{cases}v_{s}, & 0 \leq t<\frac{L_{x}}{v_{s}} \\
-v_{s}, & \frac{L_{x}}{v_{s}}+\frac{\pi}{2 w_{s}} \leq t<T_{f} \\
0, & t \geq T_{f}\end{cases} \\
& \theta_{r}(t)= \begin{cases}\pi, & 0 \leq t<\frac{L_{x}}{v_{s}} \\
\pi-w_{s}\left(t-\frac{L_{x}}{v_{s}}\right), & \frac{L_{x}}{v_{s}} \leq t<\frac{L_{x}}{v_{s}}+\frac{\pi}{2 w_{s}} \\
0, & \frac{L_{x}}{v_{s}}+\frac{\pi}{2 w_{s}} \leq t<T_{f}\end{cases} \\
& w_{r}(t)= \begin{cases}0, & 0 \leq t<\frac{L_{x}}{v_{s}} \\
-w_{s}, & \frac{L_{x}}{v_{s}} \leq t<\frac{L_{x}}{v_{s}}+\frac{\pi}{2 w_{s}} \\
0, & \frac{L_{x}}{v_{s}}+\frac{\pi}{2 w_{s}} \leq t\end{cases}
\end{aligned}
$$

where $\left(x_{s}, y_{s}\right),\left(x_{f}, y_{f}\right)$ denote, respectively, the robot's initial and terminative coordinates. In the simulation and experiment, they were set at $\left(x_{s}, y_{s}\right)=(0.43 \mathrm{~m}, 0.6 \mathrm{~m}),\left(x_{f}, y_{f}\right)=$ $(-0.57 \mathrm{~m},-0.4 \mathrm{~m})$.
TABLE II

PARAMETERS USED IN THE BACK-INTO-GARAGE SimUlation AND EXPERIMENTS

\begin{tabular}{c|c|c|c}
\hline$a_{0}$ & $k_{0}$ & $\lambda_{1}$ & $\lambda_{2}$ \\
\hline 1 & 0.10 & -2.50 & -2.80 \\
\hline
\end{tabular}

Let, $w_{f}(t)=0.1 \sin (0.1 \times t) \in S_{0.1}, \widetilde{\theta}_{r}(t)$ and $\widetilde{w}_{r}(t)$ can then be found according to (6)

$$
\left\{\begin{array}{l}
\widetilde{\theta}_{r}(t)=\frac{\pi}{2}+1-\cos \left(0.1\left(t-T_{f}+\bar{t}_{0}\right)\right) \\
\widetilde{w}_{r}(t)=0.1 \sin \left(0.1\left(t-T_{f}+\bar{t}_{0}\right)\right)
\end{array} \quad t \geq T_{f}\right.
$$

where parameter $\bar{t}_{0}=0$. Locally optimal parameters used in the experiment are given in Table II.

From Remark 4 after Theorem 3: $k_{2}(t)$ is a tuning function that smoothly transforms tracking situations into parking situations. Since the back-into-garage path only roughly satisfies Condition $\mathrm{C} 1$ ) for all $t<\pi / 2 c$, as suggested by Remark 4 , $k_{2}(t)$ should be set to a positive value before $t=\pi / 2 c$, and then changed to a zero function to perform pole placement according to Theorem 2. Details of the $k_{2}(t)$ selection for this experiment are as follows:

$$
k_{2}(t)= \begin{cases}0.125, & 0 \leq t \leq 33 \\ 0.0125 \times(43-t), & 33<t \leq 43 \\ 0, & 43<t\end{cases}
$$

Fig. 10(a)-(e) depicts results from the back-into-garage parking experiment. In the figure, the solid lines represent experimental 


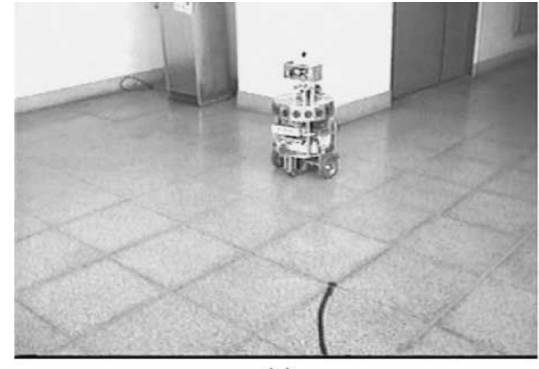

(a)

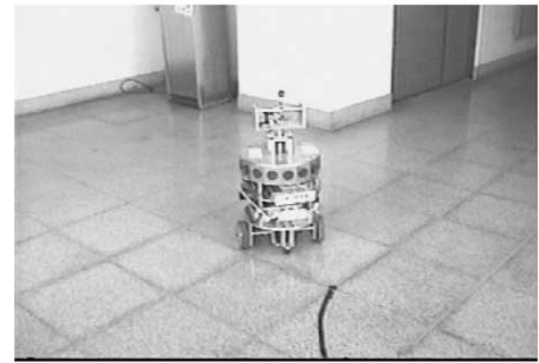

(b)

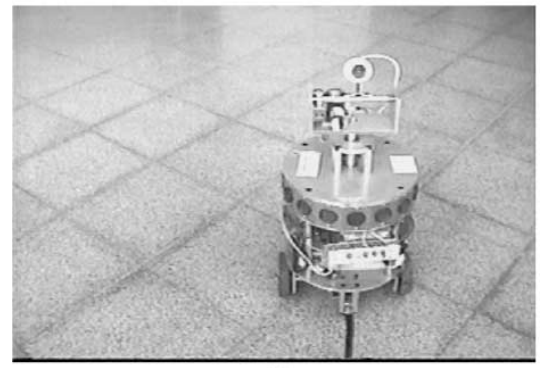

(c)

Fig. 9. Sequence of motions during the parallel parking experiment.

results using (30). For comparison, we carried out the same parking experiment with $k_{2}(t)$ as a zero function, such that

$$
k_{2}(t)=0 \text {. }
$$

The experimental results of using (31) are shown as dotted lines in Fig. 10(a)-(e). We can see that the tracking performance and convergence rate behaved satisfactorily when $k_{2}(t)$ was a positive continuous function, before $t=43 \mathrm{~s}$. The effect of the tuning function is therefore verified by these experimental results.

\section{CONCLUSION}

A novel fast parking controller for nonholonomic mobile robots based on a motion planning approach and tracking control design has been proposed and demonstrated. It solves parking problems by adding a redesigned virtual trajectory to the original trajectory. Practical experimental results verified its satisfactory convergence results. In contrast to results presented in [21], the motion planning method proposed in this paper can be extended to other higher order nonholonomic systems. An interesting application to underactuated ships can be found in [19]. Future work may focus on improving parking performance, such as in the area of robustness as it relates to uncertainty in the model and other disturbances.

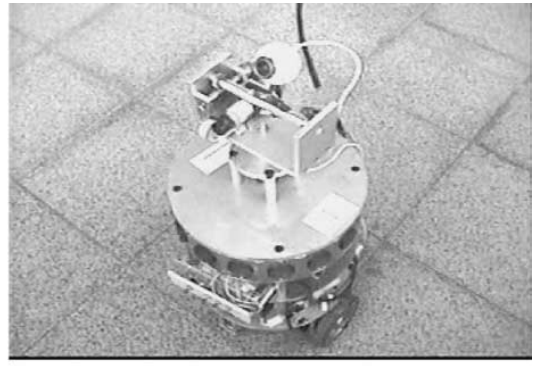

(d)

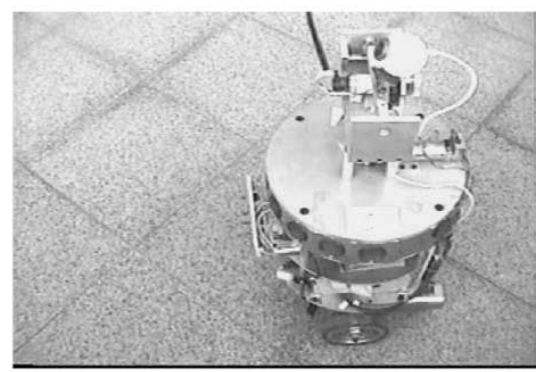

(e)

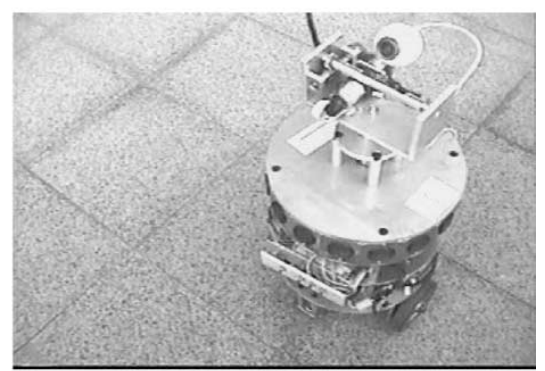

(f)

\section{APPENDIX \\ PROOF OF THEOREM 1}

A stability criterion is recalled first. Its proof can be found in [20]. Throughout this appendix, it is said that a statement $P(t)$ holds almost everywhere (a.e.) if the Lebesgue measure of the set $\{t \in[0, \infty) \mid P(t)$ is false $\}$ is zero [31].

Proposition A1: Consider the following system

$$
\dot{x}=f(t, x)
$$

where $f(t, x)$ is continuous in $x$, uniformly in $t$, and uniformly bounded on $[0, \infty] \times B_{r}$, for each $r>0$, where $B_{r}$ is an open ball centered at the origin with radius $r$. Let $V: \Re^{p} \rightarrow \Re$ be a continuously differentiable, positive-definite and proper function such that

$$
\dot{V}(t, x) \leq-W(t, x) \leq 0
$$

for all $t \geq 0$, all $x \in \Re^{p}$ where the function $W(t, x)$ is continuous in $x$, uniformly in $t$, and uniformly bounded on $[0, \infty] \times B_{r}$, for each $r>0$. The origin is then uniformly globally asymptotically stable provided that the following condition holds.

A1) For every time sequence $\left\{t_{n}\right\}$, with $\lim _{n \rightarrow \infty} t_{n}=\infty$, and every bounded continuous function $\bar{x}(t)$ that satisfies 
(a)

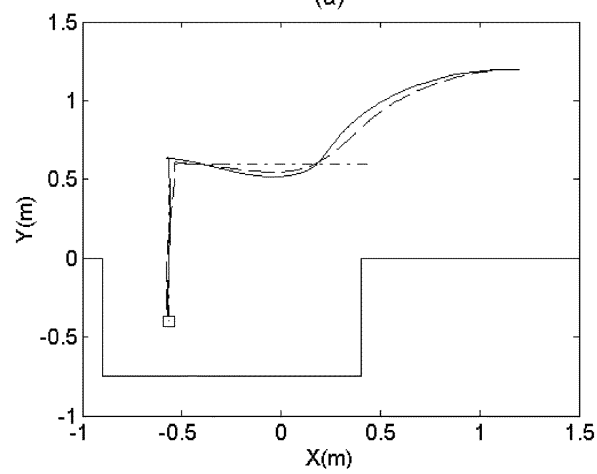

(c)
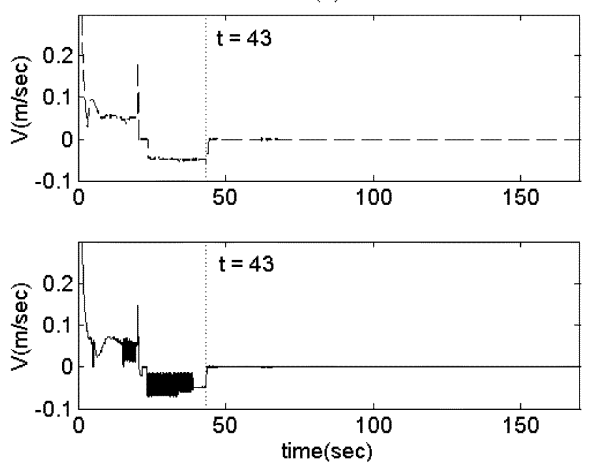

(b)
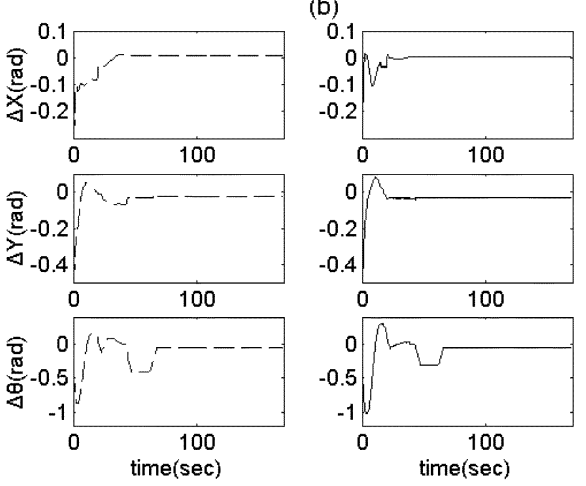

(d)
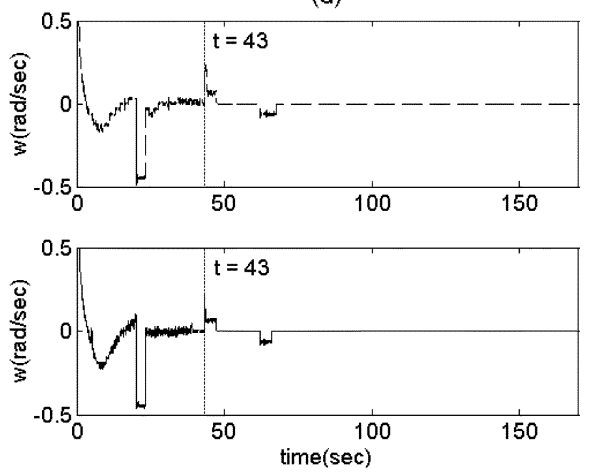

(e)

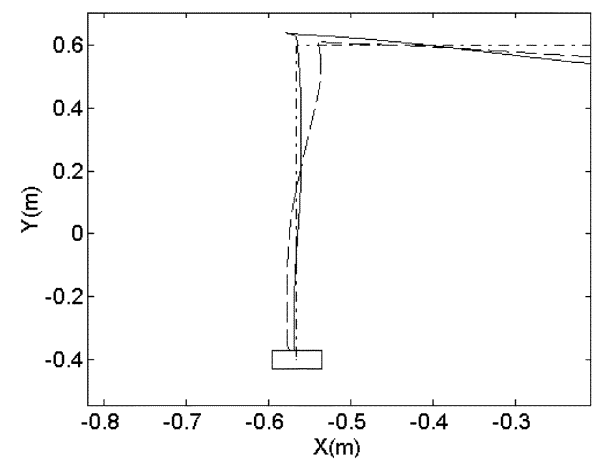

Eq. (30) Eq. $(31)$

Fig. 10. Comparison of tracking performance using two different $k_{2}(t)$ values. (a) Position variations. (b) Tracking errors. (c) Linear velocity of the center point $v_{c}$. (d) Angular velocity $w_{c}$. (e) A “zoom-in" graph of position variations.

$\lim _{n \rightarrow \infty} W\left(t+t_{n}, \bar{x}(t)\right)=0$, a. e., and the following integral equation:

$$
\bar{x}(t)=\bar{x}(0)+\lim _{n \rightarrow \infty} \int_{0}^{t} f\left(\tau+t_{n}, \bar{x}(\tau)\right) d \tau, \quad \forall t \geq 0
$$

we have $\lim _{t \rightarrow \infty} \bar{x}(t)=0$.

With the controller (9), the closed-loop system of the tracking error model (4a)-(4c) can be written into the following equations:

$$
\begin{aligned}
& \dot{x}_{0}=-\left(\frac{a_{1}}{a_{0}}\right) v_{r} \alpha\left(x_{0}\right) x_{1}-k_{0} x_{0} \\
& \dot{x}_{1}=w^{*} x_{2}+v_{r} \alpha\left(x_{0}\right) x_{0} \\
& \dot{x}_{2}=-w^{*}\left(\frac{a_{1}}{a_{2}}\right) x_{1}-\left[\left|w^{*}\right|+k_{2}\right] a_{2} x_{2}
\end{aligned}
$$

where $w^{*}=w_{r}+\left(a_{1} / a_{0}\right) v_{r} \alpha\left(x_{0}\right) x_{1}+k_{0} x_{0}$. The closed-loop system (A4)-(A6) is in the form of (A1). Since all the time functions appeared in the differential equations are bounded, it is easy to see that the function $f$ is continuous in $x$, uniformly in $t$, and uniformly bounded on $[0, \infty] \times B_{r}$, for each $r>0$. It is also seen that the Lyapunov function $V$ defined in (8) is a continuously differentiable, positive-definite and proper function that satisfies the inequality (A2) with

$$
\begin{aligned}
& W\left(t, x_{0}, x_{1}, x_{2}\right)=a_{0} k_{0} x_{0}^{2} \\
& \quad+\left[\left|w_{r}+\left(\frac{\mathrm{a}_{1}}{a_{0}}\right) v_{r} \alpha\left(x_{0}\right) x_{1}+k_{0} x_{0}\right|+k_{2}(t)\right] a_{2}^{2} x_{2}^{2}
\end{aligned}
$$

through (10). Moreover, the function $W$ is also continuous in $x$, uniformly in $t$, and uniformly bounded on $[0, \infty] \times B_{r}$, for each $r>0$. It remains to check the condition (A1) holds to guarantee the uniformly globally asymptotical stability of the origin 
in view of Proposition A1. To this end, we need the following lemma.

Lemma A1: Let $\left\{b_{n}(t)\right\}$ and $\left\{c_{n}(t)\right\}$ be two sequences of real-valued functions defined on $[0, \infty)$ with $\left|b_{n}(t)\right| \leq M_{b}$ and $\left|c_{n}(t)\right| \leq M_{c}$, for all $t \geq 0$, all $n$ in $\aleph$, and some positive constants $M_{b}$ and $M_{c}$. Suppose the following conditions hold:

$$
\begin{gathered}
\lim _{n \rightarrow \infty} \int_{0}^{t} b_{n}(\tau) d \tau=0, \quad \forall t \geq 0 . \\
\lim _{n \rightarrow \infty} \sup \left|\int_{s_{0}}^{s_{1}} c_{n}(\tau) d \tau\right|>0, \quad \text { for some } 0 \leq s_{0}<s_{1} .
\end{gathered}
$$

Let $z(t)$ be a continuous function defined on $[0, \infty)$ that satisfies

$$
z(t)=z(0)+d \lim _{n \rightarrow \infty} \int_{0}^{t} c_{n}(\tau) d \tau, \quad \forall t \geq 0
$$

for some constant $d$. Assume that the following equation

$$
z(t)\left[\lim _{n \rightarrow \infty}\left|b_{n}(t)+c_{n}(t)\right|\right]=0, \quad \text { a.e. }
$$

holds. We have then $d=0$ and $z(t) \equiv 0$.

Proof: Notice that (A8) implies that

$$
\begin{aligned}
\lim _{n \rightarrow \infty} \int_{t}^{s} b_{n}(\tau) d \tau & =\lim _{n \rightarrow \infty} \int_{0}^{s} b_{n}(\tau) d \tau-\lim _{n \rightarrow \infty} \int_{0}^{t} b_{n}(\tau) d \tau \\
& =0, \quad \forall 0 \leq t \leq s .
\end{aligned}
$$

Let us first show that $d=0$ implies $z(t) \equiv 0$. Indeed, by (A10), $z(t) \equiv z(0)$ is a constant function when $d=0$. If $z(t)$ is not a zero function, then we have $\lim _{n \rightarrow \infty}\left|b_{n}(t)+c_{n}(t)\right|=0$, a.e., in view of (A11). Consequently, by (A12) and Lebesgue dominate theorem [31]

$$
\begin{aligned}
\lim _{n \rightarrow \infty} \sup \left|\int_{s_{0}}^{s_{1}} c_{n}(\tau) d \tau\right| \leq & \left|\lim _{n \rightarrow \infty} \int_{s_{0}}^{s_{1}} b_{n}(\tau)+c_{n}(\tau) d \tau\right| \\
& +\lim _{n \rightarrow \infty}\left|-\int_{s_{0}}^{s_{1}} b_{n}(\tau) d \tau\right| \\
= & 0 .
\end{aligned}
$$

Thus, we reach a contradiction according to the inequality (A9). In the following, we show that $d=0$ and complete the proof of the lemma. We will prove it by contradiction. Suppose $d \neq 0$. From (A10), the limit $\lim _{n \rightarrow \infty} \int_{0}^{t} C_{N}(\tau) d \tau=(z(t)-z(0)) / d$ can be defined, $\forall t \geq 0$. It results in

$$
\left|\lim _{n \rightarrow \infty} \int_{s_{0}}^{s_{1}} c_{n}(\tau) d \tau\right|=\lim _{n \rightarrow \infty} \sup \left|\int_{s_{0}}^{s_{1}} c_{n}(\tau) d \tau\right|>0 .
$$

We claim that there exists a $t_{0} \in\left(s_{0}, s_{1}\right)$ such that $z\left(t_{0}\right)=0$. If the claim is false, we have $z(t) \neq 0, \forall t \in\left(s_{0}, s_{1}\right)$. Then, there exists a measure zero set $E$ such that $\lim _{n \rightarrow \infty} b_{n}(t)+c_{n}(t)=$
$0, \forall t \in\left[s_{0}, s_{1}\right]-E$ through (A11). According to (A12), this implies that

$$
\begin{aligned}
0 & =\int_{s_{0}}^{s_{1}} \lim _{n \rightarrow \infty} b_{n}(\tau)+c_{n}(\tau) d \tau \\
& =\lim _{n \rightarrow \infty} \int_{s_{0}}^{s_{1}} b_{n}(\tau)+c_{n}(\tau) d \tau \\
& =\lim _{n \rightarrow \infty} \int_{s_{0}}^{s_{1}} c_{n}(\tau) d \tau .
\end{aligned}
$$

We reach a contradiction in view of (A13). Thus, the claim is true. Particularly, we can define $\bar{s}_{0}=\inf \left\{t \in\left(s_{0}, s_{1}\right) \mid z(t)=\right.$ $0\}$ and $\bar{s}_{1}=\sup \left\{t \in\left(s_{0}, s_{1}\right) \mid z(t)=0\right\}$. By the definitions, it can be seen that $z(t) \neq 0, \forall t \in\left[s_{0}, \bar{s}_{0}\right) \cup\left(s_{1}, \bar{s}_{1}\right]$. Again from (A11), there exists a measure zero set $E$ such that $\lim _{n \rightarrow \infty} b_{n}(t) c_{n}(t)=0, \forall t \in\left\{\left[s_{0}, \bar{s}_{0}\right) \cup\left(s_{1}, \bar{s}_{1}\right]\right\}-E$ By (A12) and Lebesgue dominate theorem, this implies that the following expressions hold:

$$
\begin{aligned}
\lim _{n \rightarrow \infty} \int_{s_{0}}^{\bar{s}_{0}} c_{n}(\tau) d \tau & =\lim _{n \rightarrow \infty} \int_{s_{0}}^{\bar{s}_{0}} b_{n}(\tau)+c_{n}(\tau) d \tau \\
& =\int_{s_{0}}^{\bar{s}_{0}} \lim _{n \rightarrow \infty} b_{n}(\tau)+c_{n}(\tau) d \tau \\
& =0
\end{aligned}
$$

and

$$
\begin{aligned}
\lim _{n \rightarrow \infty} \int_{\bar{s}_{1}}^{s_{1}} c_{n}(\tau) d \tau & =\lim _{n \rightarrow \infty} \int_{\bar{s}_{1}}^{s_{1}} b_{n}(\tau)+c_{n}(\tau) d \tau \\
& =\int_{\bar{s}_{1}}^{s_{1}} \lim _{n \rightarrow \infty} b_{n}(\tau)+c_{n}(\tau) d \tau \\
& =0 .
\end{aligned}
$$

Thus, in view of (A13)

$$
\begin{aligned}
\lim _{n \rightarrow \infty} \int_{\bar{s}_{0}}^{\bar{s}_{1}} c_{n}(\tau)= & \lim _{n \rightarrow \infty} \int_{s_{0}}^{s_{1}} c_{n}(\tau) d \tau-\lim _{n \rightarrow \infty} \int_{s_{0}}^{\bar{s}_{0}} c_{n}(\tau) d \tau \\
& \lim _{n \rightarrow \infty} \int_{\bar{s}_{1}}^{s_{1}} c_{n}(\tau) d \tau \\
= & \lim _{n \rightarrow \infty} \int_{s_{0}}^{s_{1}} c_{n}(\tau) d \tau \neq 0 .
\end{aligned}
$$

Since $z(t)$ is a continuous function, it can be seen that $z\left(\bar{s}_{0}\right)=$ $z\left(\bar{s}_{1}\right)=0$ by the definitions. Consequently, we have $0=$ $z\left(\bar{s}_{1}\right)-z\left(\bar{s}_{0}\right)=d \lim _{\mathrm{n} \rightarrow \infty} \int_{\bar{s}_{0}}^{\bar{s}_{1}} c(\tau) d \tau$, and hence, $d=0$ in view of (A14). We reach another contradiction. It completes the proof. $\square$

Now, let us check condition (A1) by employing Lemma A1. Let $\left\{t_{n}\right\}$ be any time sequence with $\lim _{n \rightarrow \infty} t_{n}=\infty$ and $\left(\bar{x}_{0}(t)\right.$, $\left.\bar{x}_{1}(t), \bar{x}_{2}(t)\right)$ be any bounded continuous functions such that 
$\lim _{n \rightarrow \infty} W\left(t+t_{n}, \bar{x}_{0}(t), \bar{x}_{1}(t), \bar{x}_{2}(t)\right)=0$, a. e., and the following equations hold:

$$
\begin{aligned}
\bar{x}_{0}(t)= & \bar{x}_{0}(0)-\lim _{n \rightarrow \infty} \int_{0}^{t} b_{n}(\tau) d \tau \\
\bar{x}_{1}(t)= & \bar{x}_{1}(0)+\lim _{n \rightarrow \infty} \int_{0}^{t}\left[w_{r}\left(\tau+t_{n}\right)+b_{n}(\tau)\right] \bar{x}_{2}(\tau) \\
& +v_{r}\left(\tau+t_{n}\right) \alpha\left(\bar{x}_{0}(\tau)\right) \bar{x}_{0}(\tau) d \tau \\
\bar{x}_{2}(t)= & \bar{x}_{2}(0)-\lim _{n \rightarrow \infty} \int_{0}^{t}\left[w_{r}\left(\tau+t_{n}\right)+b_{n}(\tau)\right]\left(\frac{a_{1}}{a_{2}}\right) \bar{x}_{1}(\tau) \\
& -\left[\left|w_{r}\left(\tau+t_{n}\right)+b_{n}(\tau)\right|+k\left(\tau+t_{n}\right)\right] \\
& \times a_{2} \bar{x}_{2}(\tau) d \tau
\end{aligned}
$$

where

$b_{n}(t)=\left(\frac{a_{1}}{a_{0}}\right) v_{r}\left(t+t_{n}\right) \alpha\left(\bar{x}_{0}(t)\right) \bar{x}_{1}(t)+k_{0} \bar{x}_{0}(t)$

$\forall t \geq 0, \quad \forall n \in \aleph$.

Notice that the equation $\lim _{n \rightarrow \infty} W\left(t+t_{n}, \bar{x}_{0}(t), \bar{x}_{1}(t), \bar{x}_{2}(t)\right)=$ 0 , a. e., is equivalently to saying that

$$
\begin{aligned}
& \bar{x}_{0}(t)=0, \quad \text { a.e. } \\
& \bar{x}_{2}(t) \lim _{n \rightarrow \infty}\left|w_{r}\left(t+t_{n}\right)+b_{n}(t)\right|=0, \quad \text { a.e. }
\end{aligned}
$$

and

$$
\bar{x}_{2}(t) \lim _{n \rightarrow \infty} k_{2}\left(t+t_{n}\right)=0, \quad \text { a.e.. }
$$

Since $\bar{x}_{0}(t)$ is a continuous function, (A18) implies that $\bar{x}_{0}(t)$ is a zero function [31]. Thus, (A15) is reduced to the (A8), i.e., the condition (i) in Lemma A1 holds. Moreover, (A16) is also reduced to

$$
\begin{aligned}
\bar{x}_{1}(t) & =\bar{x}_{1}(0)+\int_{0}^{t} \lim _{n \rightarrow \infty}\left[w_{r}\left(\tau+t_{n}\right)+b_{n}(\tau)\right] \bar{x}_{2}(\tau) d \tau \\
& =\bar{x}_{1}(0), \quad \forall t \geq 0
\end{aligned}
$$

by using Lebesgue dominate theorem [31] and (A19). In particular, $\bar{x}_{1}(t)$ is a constant function. Let $d=-\bar{x}_{1}(0) a_{1} / a_{2}$, $z(t)=\bar{x}_{2}(t)$ and $c_{n}(t)=w_{r}\left(t+t_{n}\right), \forall t \geq 0, \forall n \in \aleph$. (A19) is then equivalent to (A11) and (A17) is reduced to (A10) by using the (A8), (A19), and (A20). Now, let us verify condition ii) in Lemma $\mathrm{A} 1$ by assuming the Condition $\mathrm{C} 2$ ). If Condition C2) holds, there exist three positive constants $T_{0}, r$ and $\delta$ so that

$$
\left|\int_{\boldsymbol{s}_{n}}^{\boldsymbol{s}_{n}+\delta} c_{n}(\tau) d \tau\right|>r
$$

for some sequence $\left\{s_{n}\right\}$ with $0 \leq s_{n} \leq T_{0}$. Since $\left\{s_{n}\right\}$ is contained in the compact set $\left[0, T_{0}\right]$, there exists a subsequence $\left\{s_{n_{k}}\right\}$ of $\left\{s_{n}\right\}$ converging to a point $s_{0} \geq 0$. This results in

$\lim _{n \rightarrow \infty} \sup \left|\int_{s_{0}}^{s_{0}+\delta} c_{n}(\tau) d \tau\right| \geq \lim _{k \rightarrow \infty} \sup \left|\int_{s_{n_{k}}}^{s_{n_{k}}+\delta} c_{n_{k}}(\tau) d \tau\right| \geq r>0$.

Thus, ii) in Lemma A1 holds under Condition C2). It can be concluded that $\bar{x}_{0}(t) \equiv 0, \bar{x}_{1}(t) \equiv 0$ and $\bar{x}_{2}(t) \equiv 0$ in view of Lemma A1. Thus, condition (A1) holds and the origin of the closed-loop system is uniformly globally asymptotically stable by using Proposition A1 and Condition C2). On the other hand, assume that $k_{2}(t) k_{1} \geq 0, \forall t \geq 0$. Equation (A20) implies that $\bar{x}_{2}(t)=0$, a.e. Since $\bar{x}_{2}(t)$ is a continuous function, this implies that $\bar{x}_{2}(t)$ is a zero function. Notice that the (A15) is reduced to

$$
\bar{x}_{1}(0) \lim _{n \rightarrow \infty} \int_{0}^{t} v_{r}\left(\tau+t_{n}\right) d \tau=0 \quad, \forall t \geq 0
$$

due to the fact that $\bar{x}_{0}(t)$ is a zero function, $\bar{x}_{1}(t)$ is a constant function and $\alpha(0)=1$. Using a similar argument as in the previous discussion, condition (C1) implies that there exist four positive constants $T_{0}, r, \delta, s_{0} \geq 0$ so that

$\lim _{n \rightarrow \infty} \sup \left|\int_{s_{0}}^{s_{0}+\delta} v_{r}\left(\tau+t_{n}\right) d \tau\right|+\lim _{n \rightarrow \infty} \sup \left|\int_{s_{0}}^{s_{0}+\delta} c_{n}\left(\tau+t_{n}\right) d \tau\right| \geq r$.

If $\bar{x}_{1}(0) \neq 0, \lim _{n \rightarrow \infty} \int_{0}^{t} v_{r}\left(\tau+t_{n}\right) d \tau=0, \forall t \geq 0$, by (A22). This implies that (ii) in Lemma A1 holds and again $\bar{x}_{1}(0)=0$ by Lemma A1. We reach a contradiction. Thus, $\bar{x}_{1}(0)=0$ and we conclude that $\bar{x}_{0}(t) \equiv 0, \bar{x}_{1}(t) \equiv 0$, and $\bar{x}_{2}(t) \equiv 0$ under Condition C1) and the assumption $k_{2}(t) \geq k_{1}>0, \forall t \geq 0$. Again, condition (A1) holds and the origin of the closed-loop system is uniformly globally asymptotically stable via Proposition A1. A similar argument can be applied to the linearized closed-loop system, that can also be described by (A4)-(A6) with $\alpha \equiv 1$ and $w^{*} \equiv w_{r}(t)$. Consequently, it can be concluded that the origin of the linearized system is also uniformly globally asymptotically stable. Hence, the origin of the closed-loop system is locally exponentially stable [16]. This completes the proof.

\section{ACKNOWLEDGMENT}

The authors would like to thank C.-C. Chien and K.-M. Yan of the Intelligent System Control Integration Lab, National Chiao Tung University, for their assistance in construction of the mobile robot. The authors would also like to thank the Associate Editor, Prof. K. L. Moore, and the anonymous referees for their constructive comments and suggestions.

\section{REFERENCES}

[1] A. Astolfi and W. Schaufelberger, "State and output feedback stabilization of multiple chained systems with discontinuous control," in Proc. IEEE 35th Conf. Decision Control, Japan, 1996, pp. 1443-1448.

[2] A. M. Bloch, M. Reyhanoglu, and N. H. McClamroch, "Control and stabilizability of nonholonomic dynamic systems," IEEE Trans. Automat. Contr., vol. 37, pp. 1746-1757, Nov. 1992.

[3] R. W. Brockett, "Asymptotic stability and feedback stabilization," in Differential Geometric Control Theory, R. W. Brockett, R. S. Millman, and H. H. Sussmann, Eds. Cambridge, MA: Birkhauser, 1983, pp. $181-191$.

[4] C. C. de Wit, H. Berghuis, and H. Nijmeijer, "Practical stabilization of nonlinear systems in chained form," in Proc. IEEE 33th Conf. Decision Contol, Lake Buena Vista, FL, 1994, pp. 3475-3480.

[5] C. de Wit Canudas, B. Siciliano, and G. Bastin, Eds., Theory of Robot Control. London, U.K.: Springer-Verlag, 1996.

[6] C. C. de Wit and O. J. Sordalen, "Exponential stabilization of mobile robots with nonholonomic constraints," IEEE Trans. Automat. Contr., vol. 37, pp. 1791-1797, Nov. 1992. 
[7] C. C. Chang and K. T. Song, "Environment prediction for a mobile robot in a dynamic environment," IEEE Trans. Robot. Automat., vol. 13, pp. 862-872, Dec. 1997.

[8] C. T. Chen, Introduction to Linear System Theory. New York: Holt, Rinehart and Winston, 1986.

[9] J. M. Coron, "Global asymptotic stabilization for controllable systems without drift," Math. Contr., Signals, Syst., vol. 5, pp. 295-312, 1992.

[10] W. E. Dixon, D. M. Dawson, E. Zergeroglu, and F. Zhang, "Robust tracking and regulation control for mobile robots," Int. J. Robust Nonlinear Contr., vol. 10, pp. 199-216, 2000.

[11] K. D. Do, Z. P. Jiang, and J. Pan, "A universal saturation controller design for mobile robots," in Proc. IEEE 41st Conf. Decision Control, Las Vegas, NV, 2002, pp. 2044-2049.

[12] — "Universal controllers for stabilization and tracking of underactuated ships," Syst. Contr. Lett., vol. 47, pp. 299-317, 2002.

[13] J. P. Hespanha, D. Liberzon, and A. S. Morse, "Logic-based switching control of a nonholonomic system with parametric modeling uncertainty," Syst. Contr. Lett., vol. 38, pp. 167-177, 1999.

[14] Z. P. Jiang, "Robust exponential regulation of nonholonomic systems with uncertainties," Automatica, vol. 36, pp. 189-209, 2000

[15] Z. P. Jiang and H. Nijmeijer, "Tracking control of mobile robots: a case study in backstepping," Automatica, vol. 33, pp. 1393-1399, 1997.

[16] H. K. Khalil, Nonlinear Systems. New York: Macmillian, 1992.

[17] B. M. Kim and P. Tsiotras, "Controllers for unicycle-type wheeled robots: theoretical results and experimental validation," IEEE Trans. Robot. Automat., vol. 18, pp. 294-307, 2002.

[18] T. C. Lee, "Practical stabilization for nonholonomic chained systems with fast convergence, pole-placement and robustness," in Proc. IEEE Int. Conf. Robotics and Automation, Washington DC, 2002, pp. 3534-3539.

[19] T. C. Lee, Tracking of Underactuated Ships via a Relaxed PE Condition, to be published.

[20] T. C. Lee and B. S. Chen, "A general stability criterion for time-varying systems using a modified detectability condition," IEEE Trans. Automat. Contr., vol. 47, pp. 797-802, May 2002.

[21] T. C. Lee, K. T. Song, C. H. Lee, and C. C. Teng, "Tracking control of unicycle-modeled mobile robots using a saturation feedback controller," IEEE Trans. Contr. Syst. Technol., vol. 9, pp. 305-318, Mar. 2001.

[22] D. A. Lizarraga, P. Morin, and C. Samson, "Non-robustness of continuous homogeneous stabilizers for affine control systems," in Proc. IEEE 38th Conf. Decision Control, Phoenix, AZ, 1999, pp. 855-860.

[23] R. T. M'Closkey and R. M. Murray, "Exponential stabilization of driftless control systems using homogeneous feedback," IEEE Trans. Automat. Contr., vol. 42, pp. 614-628, May 1997.

[24] P. Morin and C. Samson, "Control of nonlinear chained systems: from Routh-Hurwitz stability criterion to time-varying exponential stabilizers," IEEE Trans. Automat. Contr., vol. 45, pp. 141-146, Jan. 2000.

[25] —, "Robust stabilization of driftless systems with hybrid open-loop/feedback control," in Proc. ACC, Chicago, IL, 2000, pp. 3929-3933.

[26] - "Practical stabilization of driftless systems on Lie groups," in Proc IEEE 41st Conf. Decision Control, Las Vegas, Nevada, 2002, pp. 4272-4277.

[27] R. M. Murray and S. S. Sastry, "Nonholonomic motion planning: steering using sinusoids," IEEE Trans. Automat. Contr., vol. 38, pp. 700-716, May 1993.

[28] K. S. Narendra and A. M. Annaswamy, Stable Adaptive Systems. Englewood Cliffs, NJ: Prentice-Hall, 1989.

[29] I. E. Paromtchik and C. Laugier, "Autonomous parallel parking of a nonholonomic vehicle," in Proc. IEEE Intelligent Vehicles Symp., Tokyo, Japan, 1996, pp. 13-18.

[30] J. B. Pomet, "Explicit design of time-varying stabilizing control laws for a class of controllable systems without drift," Syst. Control Lett., vol. 18, pp. 467-473, 1992
[31] W. Rudin, Real and Complex Analysis. New York: McGraw-Hill, 1987.

[32] C. Samson, "Control of chained systems application to path following and time-varying point-stabilization of mobile robots," IEEE Trans. Automat. Contr., vol. 40, pp. 64-77, Jan. 1995.

[33] C. Samson and K. Ait-Abderrahim, "Feedback control of a nonholonomic wheeled cart in Cartesian space," in Proc. IEEE Int. Conf. Robotics and Automation, Sacramento, CA, 1991, pp. 1136-1141.

[34] K. T. Song and C. E. Li, "Tracking control of a fee ranging automatic guided vehicle," Control Eng. Practice, vol. 1, no. 1, pp. 163-169, 1993.

[35] K. T. Song and L. H. Sheen, "Heuristic fuzzy-neuro network and its application to reactive navigation of a mobile robot," Fuzzy Sets Syst., vol. 110 , no. 3, pp. 331-340, 2000

[36] O. J. Sordalen and O. Egeland, "Exponential stabilization of nonholonomic chained systems," IEEE Trans. Automat. Contr., vol. 40, pp. 35-49, Jan. 1995.

[37] M. Vidyasagar, Nonlinear Systems Analysis. Englewood Cliffs, NJ: Prentice-Hall, 1993.

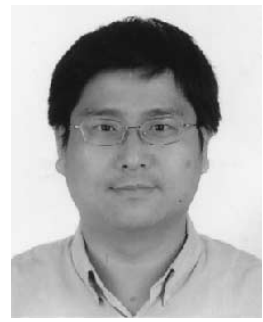

Ti-Chung Lee was born in Taiwan, R.O.C., in 1966. $\mathrm{He}$ received the M.S. degree in mathematics and the $\mathrm{Ph} . \mathrm{D}$. degree in electrical engineering from the National Tsing Hua University, Hsinchu, Taiwan, in 1990 and 1995, respectively.

$\mathrm{He}$ is currently an Associate Professor in the Department of Electrical Engineering, Ming Hsin University of Science and Technology, Hsinchu. His main research interests are nonlinear control systems, nonholonomic systems, and robot control.

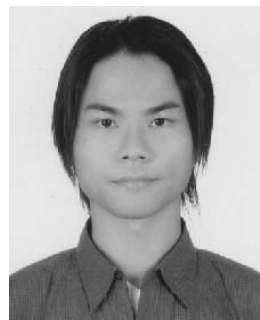

Chi-Yi Tsai was born in Kaohsiung, Taiwan, R.O.C., in 1978. He received the B.S. and M.S. degrees in electrical engineering from National Yunlin Technology University, Yunlin, Taiwan, in 2000 and 2002, respectively. He is currently working toward the Ph.D. degree in electrical and control engineering at the National Chiao Tung University, Hsinchu, Taiwan.

His research interests include visual motion planning of mobile robots, visual servoing, and computer vision.

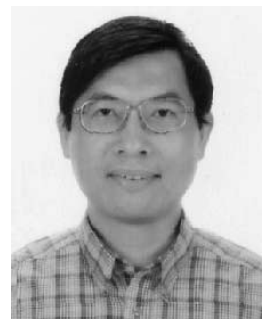

Kai-Tai Song (A'91) was born in Taipei, Taiwan, R.O.C., in 1957. He received the B.S. degree in power mechanical engineering from National Tsing Hua University, Hsinchu, Taiwan, in 1979, and the $\mathrm{Ph} . \mathrm{D}$. degree in mechanical engineering from the Katholieke Universiteit Leuven, Belgium, in 1989.

He was with the Chung Shan Institute of Science and Technology from 1981 to 1984 . Since 1989, he has been on the faculty and is currently a Professor in the Department of Electrical and Control Engineering, National Chiao Tung University, Hsinchu, Taiwan. His areas of research interest include mobile robots, image processing, visual tracking, sensing and perception, embedded systems, intelligent system control integration, and mechatronics. 